\title{
Analysis of explosion risk factor potential on coal reclaim tunnel facilities by modified analytical hierarchy process
}

\author{
Nuhindro Priagung Widodo ${ }^{1}\left[\right.$ Budi Sulistianto $^{1} \cdot$ Ahmad Ihsan $^{1}$
}

Received: 13 April 2018/Revised: 26 June 2018/Accepted: 30 August 2018/Published online: 14 September 2018

(C) The Author(s) 2018

\begin{abstract}
This study focused on developing a risk assessment method for explosion at a coal reclaim tunnel (CRT) facility. The method was developed based on an analytical hierarchy process (AHP), which is an expert system that quantifies the factors of explosion incidents, based on events and hierarchies. In this paper, the proposed model was modification from original AHP model, specifically modifying the structure from "alternative's results" to "total risk-rating's results". The total risk-rating is obtained by summing up risk-rating of each factor, where the risk-rating is a multiplication product of the risk value by the AHP weighted value. To support decision-making using the expert system, data on the real conditions of the CRT were collected and analyzed. A physical modeling of the CRT with laboratory-scale experiments was carried out to show the impact of a ventilation system in CRT on diluting the methane gas and coal dust, in order to support the quantification of AHP risk value. The criteria to evaluate the risk of explosion was constructed from six components that are: fuel, oxygen, ignition, confinement, dispersion, and monitoring system. Those components had fifty-two factors that serve as sub-components (root causes). The main causes of explosion in CRT were found to be: mechanical ventilation failure and abnormal ventilation, breakdown of monitoring system, and coal spontaneous-combustion. Assessments of two CRT facilities at Mine A and Mine B were carried out as a case study in order to check the reliability of the developed AHP method. The results showed that the risk rating of Mine A was classified as high and Mine B was classified as medium, which is in a good agreement with the site conditions.
\end{abstract}

Keywords Risk - Explosion - Coal reclaim tunnels - Analytical hierarchy process

\section{Introduction}

Reclaim tunnels are usually constructed underneath the coal stockpiles area near the port and are equipped with a conveyor belt to transport coal continuously from stockpile to a coal barge or to a coal carrier. In coal reclaim tunnel, there is a risk of fire and explosion because the explosive methane gas and coal dust could be present in the tunnel as consequences of coal transportation operations.

Nuhindro Priagung Widodo

agung@mining.itb.ac.id

1 Mining Engineering Group, Faculty of Mining and Petroleum Engineering, Institut Teknologi Bandung, Jl. Ganesha No. 10, Bandung 40132, Indonesia
According to the risk assessment matrix of the Australian and New Zealand risk management standards AS/ NZS 4360:2004 (Ristić 2013), risk level is defined by comparing the likelihood and potential consequences of accident event and can be classified into four levels: extreme risk, high risk, medium risk, and low risk. The CRT explosion can be classified as an extreme risk level (which means a detailed action plan is required) because the likelihood of accident is ranked between "possible" and "almost certain". Moreover, the potential consequences can be rated "catastrophic" (Smith and Du Plessis 1999). This risk has to be reduced from extreme risk to medium or low risk that can be managed by conducting mitigation plan or risk control. In order to control the risk effectively, factors that contribute to the risk must be 
understood and the relationships of the factors have to be quantified.

Significant research has been conducted on fire and explosion caused by methane gas and coal-dust in underground coal mines and coal stockpiles (Brooks et al. 1988; Smith and Du Plessis 1999; Kissell et al. 2007; Brune et al. 2007; Yuan and Smith 2012; Chalmers 2013). However, only a few of articles mention explosion in CRT. One of these articles appeared in "Guidelines of Safety requirements for coal stockpiles and reclaim tunnels" (Mine Safety Operations Branch New South Wales Australia Trade \& Investment 2013), which pointed out that the CRT hazards are related to: people accessing a reclaim tunnel, tunnel blockages impeding means of egress, atmospheric contamination, electricity, fire, explosion, flooding, conveyor failure, draw down equipment failure, airborne dust, and poor maintenance on feeders and valves. The prevention and handling of explosion risk at a reclaim tunnel facility has been explained in the literature, but the quantitative risk from the combination of those hazards has not been described specifically.

This study aims to develop a risk management method at a coal reclaim tunnel facility using the principles of the analytical hierarchy process or AHP (Merna and Al-Thani 2008). The method was chosen because the explosion processes at a reclaim tunnel facility are triggered by a number of events and consist multiple hierarchies, each factor of which can be quantified by AHP. The developed AHP model was a modification of original model (Saaty 2008) that is modified by changing the ending of the AHP structure from "alternative results" to "risk- score results". Moreover, the developed AHP model also refer to the one presented by Lang and Fu-Bao (2010), who developed a similar method for assessing the risk of spontaneous combustion in a coal seam.

In this present research, experimentation using a physical model of a CRT on a laboratory scale was carried out in order to study the effect of the ventilation system in the CRT. Furthermore, the study case using modified AHP method has been conducted in CRT facilities with different conditions, in order to check the reliability of the developed AHP method.

\subsection{Explosion risk}

An explosion in underground facilities or tunnels is one of the most feared mining accidents. The explosion is very dangerous to the miners life and all facilities underground due to its very high released energy and the difficulty in preventing and controlling it, as the cause of explosion is very complex and the location is very difficult to access. Data collected by the Mine Safety and Health Administration (MSHA) in the United States presented by Brnich and Kowalski-Trakofler (2010) in Table 1 show that the most frequent accidents in underground coal mining are explosion and fire related to methane gas as strata gas and coal dust resulting from mining operations.

Five conditions are required for an explosion: fuel, heat, oxygen, mixing (suspension), and isolated space (confinement). The first three factors are called the fire triangle. According to Stephan (1998), the pressure and speed of the explosion are strongly influenced by the suspension factor, whereas the confinement factor serves to maintain the concentration of dust at the Lower Explosive Limit (LEL) and to confine energy from the explosion.

\subsection{Coal reclaim tunnel}

A coal reclaim tunnel is facility located underneath the coal stockpile (as illustrated in Fig. 1) that serves as a transfer point for coal from the stockpile to other areas. Coal from the stockpile will be transferred onto the conveyor belt through the feeder, and then the conveyor brings the coal to the destination, such as a coal barge or vessel. There is some equipment inside the CRT, including the conveyor, coal feeder, jet fan and others. Dimensions of the CRT vary depending on the size of the coal stockpile and conveyor belt.

According to the Denton (2004), the conditions that trigger the occurrence of explosion in CRT are as follows: coal dust that is passed through the coal feeder; methane gas is released from coal; sparks from an electrical motor such as in jet fan, conveyor belt motor, lamp and so forth; heat from a moving conveyor; conditions of the confined space (confinement), and so on. Other factors also contribute to explosion in CRT, including heat from coal spontaneous combustion, presence of $\mathrm{CO}$ gas (which is a combustible gas from incomplete combustion), and insufficient ventilation system.

Table 1 Number of underground coal mine worker fatalities by type of disaster in United States, 1900-2008 (Brnich and KowalskiTrakofler 2010)

\begin{tabular}{lrc}
\hline Type of incident & Number of events & Percentage $(\%)$ \\
\hline Explosion & 420 & 81.7 \\
Fire & 35 & 6.8 \\
Haulage & 21 & 4.1 \\
Ground fall/bump & 14 & 2.7 \\
Inundation & 7 & 1.4 \\
Other & 17 & 3.3 \\
\hline
\end{tabular}




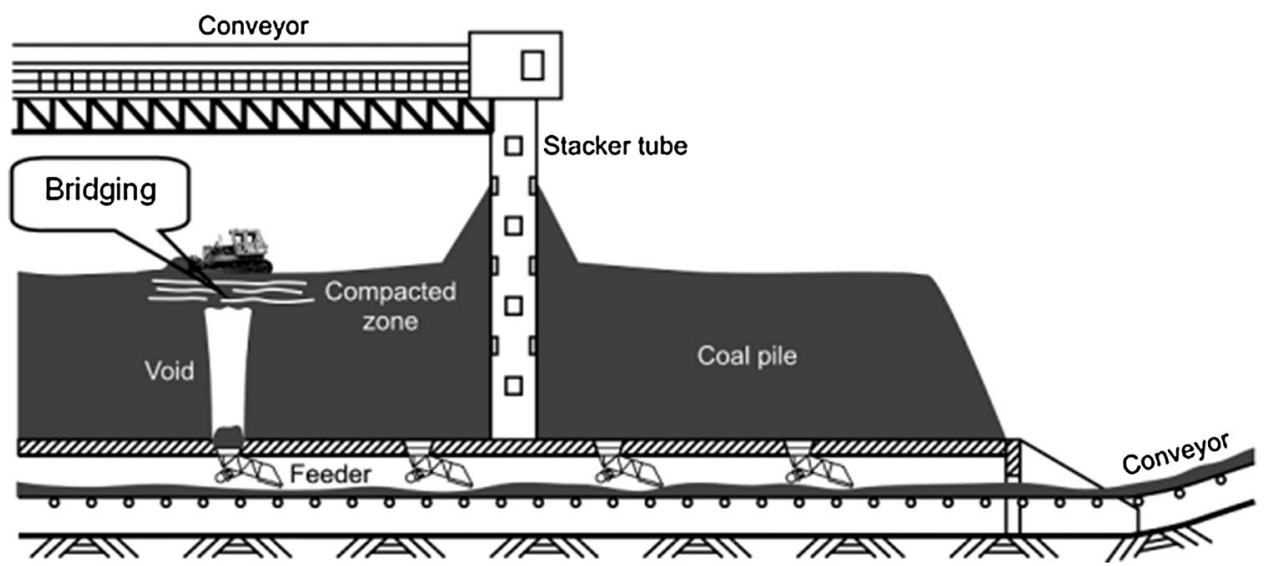

Fig. 1 Design example of stockpile and reclaim tunnel (NSW Guidelines 2013)

\subsection{Modified AHP}

According to Merna and Al-Thani (2008), some of the preferred methods used to find the root cause of risk are: hazard and operability study, fault tree analysis, what-if analysis, and checklist. However, those are not suitable to understand the weighted value of a root problem in terms of the event. The AHP developed by Saaty (1980) use pairwise comparisons and relies on the judgements of experts to derive priority scales. The AHP can quantify each factors that contributes to the risk. The steps to perform the analysis with AHP are as follows (Saaty 2008):

(1) define the problem and determine the kind of knowledge required,

(2) establish the decision hierarchy (goal of the decision, criteria on which subsequent elements depend, and alternatives),

(3) weigh the priorities, and continue this process of weighing and adding, until the final priorities are obtained.

The AHP structure developed in this research is a modification of the second and third steps of the original structure listed above and the final priorities or alternatives are not used as a conclusion in the modified AHP. The modified AHP structure can be seen in Fig. 2. Hierarchy I is the "risk", Hierarchy II is the "main factors" that contribute to the risk, and Hierarchy III is the "cause factors" that contribute to each main factors.

The modified AHP is conducted in several stages as follows (Fig. 3):

\section{(a) Determine the cause of the explosion factor in a CRT.}

The risk of explosion in CRT has two main factors, namely internal and external factors. Internal factors come from natural conditions, such as: coal dust, methane gas, spontaneous combustion propensity, and so forth. External factors are derived from engineering design and confined space condition, insufficient airflow quantity, the presence of external triggers, and so on. These factors are described in the modified AHP structure.

(b) Calculate the weighted value of each factor.

The weighted value is derived from the expert assessment, which is then processed using Super Decisions software (RC1 2016).

(c) Determine the parameters of the risk level for each factor.

Parameters are derived from some references and are also derived from site assessments.

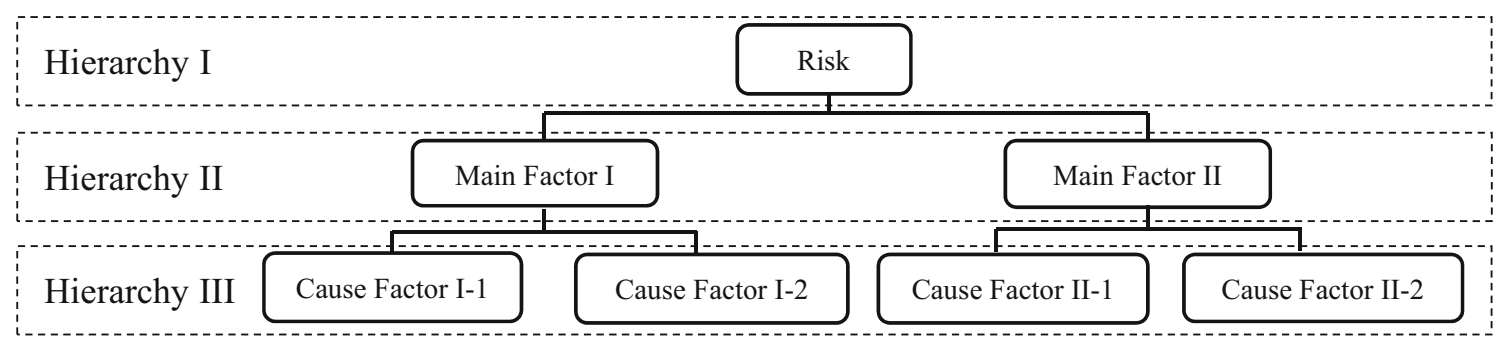

Fig. 2 The modified AHP structure (modification from Saaty 2008) 


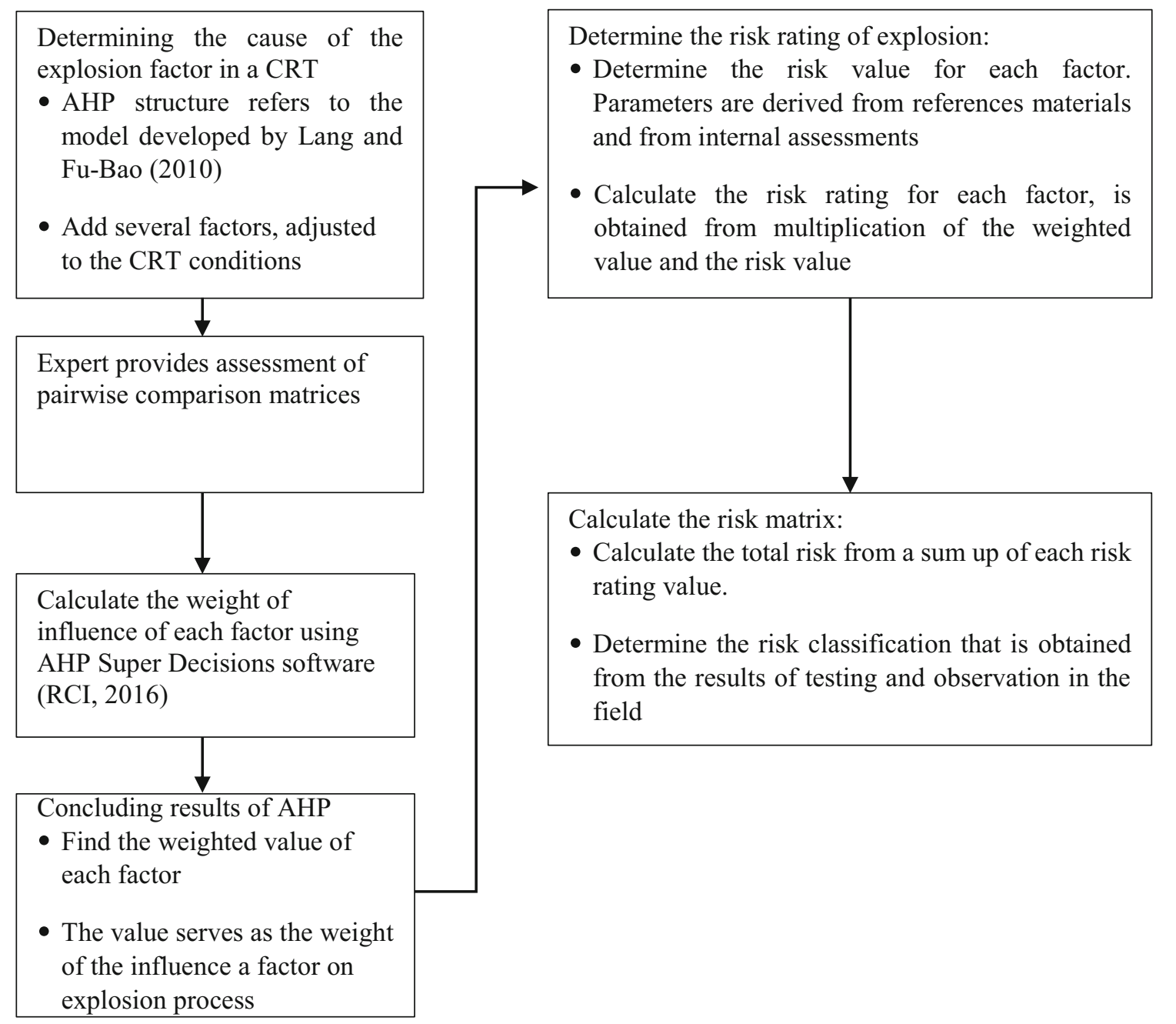

Fig. 3 Research stages to develop modified AHP model

(d) Calculation of risk matrix, include:

- Calculate the total risk from a sum up of each risk rating value.

- Determine the risk classification that is obtained from the results of testing and observation in the field

In modified AHP model, the highest hierarchy (Hierarchy I) is the event of explosion. The second hierarchy is the factors causing the explosion. The second hierarchy is the factors causing the explosion. The hierarchy consists of six factors: fuel, oxygen, ignition, confinement, dispersion and monitoring system. These six factors are divided into 52 cause factors that are expressed in the lower hierarchy, as shown in Fig. 4.

Based on the assessment of pairwise comparison matrices and analysis by Super Decision, the weighted value results are shown in Table 2 . The results show that spontaneous combustion factor is the largest contributor to explosion in CRT. The first ten factors are the dominant factors that contribute to 55.86\% (Mechanical Ventilation, Monitoring System, and Coal spontaneous combustion) of the explosions risk. The weighted value for each factor have to combined with values of the factor that represent individual risk of each factor, and the summation of all factors is then analyzed to estimate the CRT explosion risk potential.

The risk value $(R V)$ is a semi-quantitative value that combine the quantitative value from technical data and qualitative value from expert judgement based on site conditions, which then RV will be applied to represent individual parameter risk of CRT explosion. The risk rating of each factors $\left(R R_{i}\right)$ then is calculated using Eq. (1), which is multiplication of " 100 " as a constant value, weighted value for each factor $\left(C_{i}\right)$ as described in Table 2 , and risk values for each factor $\left(R V_{i}\right)$. The formula used to determine risk rating for each factor is as follows: 


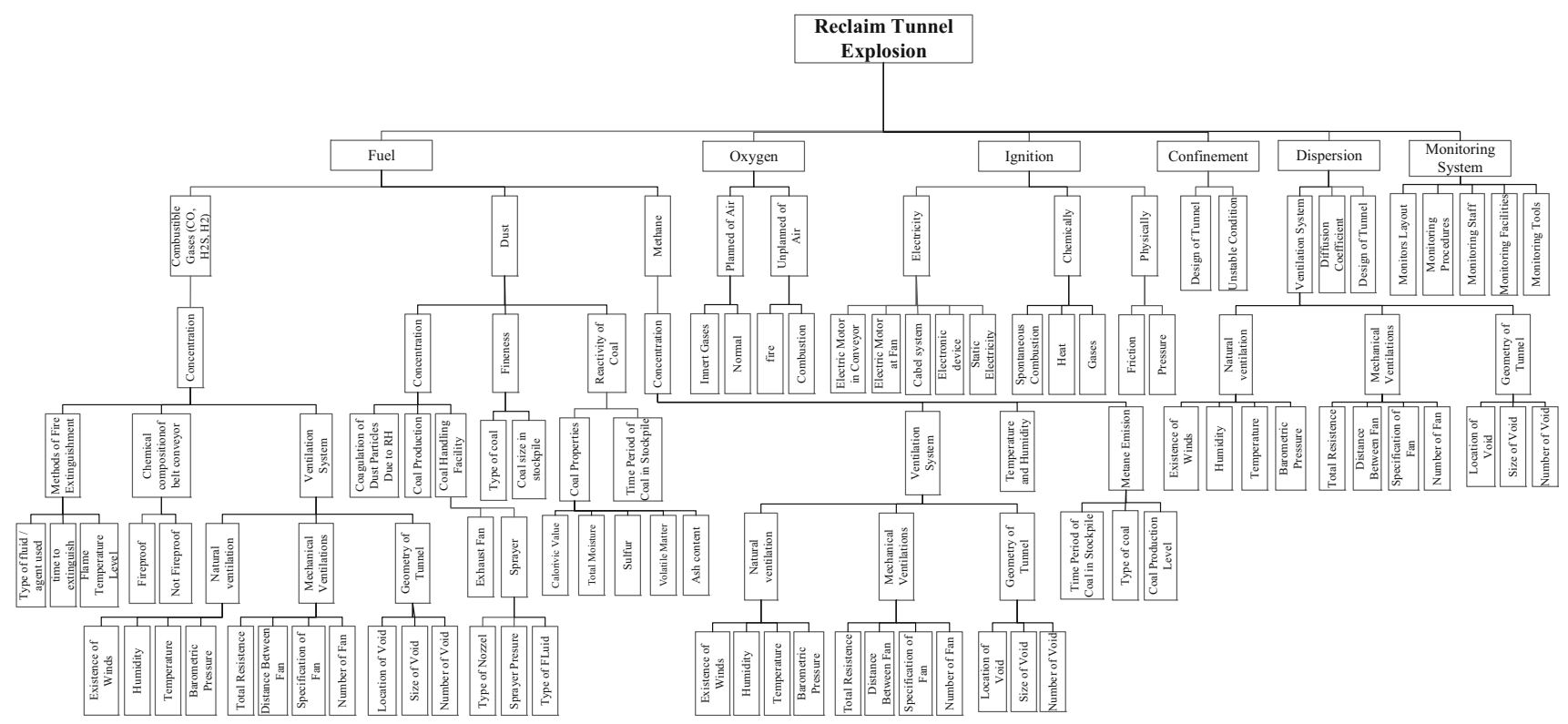

Fig. 4 Modified AHP structure for explosion root cause in CRT

$R R_{i}=100 \times C_{i} \times R V_{i}$

where a constant value of 100 is used to create a sufficient range for risk classification, $R R_{i}=$ Risk rating for factor $i$, $C_{i}=$ Weighted value for factor $i, R V_{i}=$ risk value for factor $i, i=$ code of factor.

After calculating $R R$ for each factor, then the $R R$ of all factors must be summed to get the total risk rating (TRR), as follows:

$T R R=\sum_{i}^{n} R R$

where $R R=$ Risk rating, $T R R=$ Total risk rating, $i=$ code of factor " $i$ ", $n=$ code of factor " $n$ "

The authors classify the level of TRR into five groups/classes that is from Class 1 to Class 5 (with interval of TRR is 100 point), where Class 5 is classified as "very high risk" and Class 1 is classified as "very low risk", as shown in Table 3.

The ventilation system parameters are a cause factor that is mainly related with the concentration of methane gas and coal dust (as the main fuel components) in CRT explosions. To investigate this factor, the laboratory physical model (Figs. 5, 6) has been developed at the Center of Research Excellence in Underground Mining and Mine Safety of the Institut Teknologi Bandung, Indonesia (CoRE UMMS). The experiments were carried out to investigate the fan system configuration that would optimally dilute and remove dangerous gases and coal dust in CRT by measuring the concentration-time curves of methane gas that injected in CRT's physical model.
The physical model was constructed of acrylic (methyl methacrylate monomer) $5 \mathrm{~mm}$ in thickness, and has a cross-sectional area of $40 \mathrm{~cm} \times 40 \mathrm{~cm}$, and a length of $6 \mathrm{~m}$, which is a scaled down of the real CRT at mine site (1:10 of cross-sectional area and 1:35 of length). The model has two rectangular obstructions that represented coal feeders in CRT. Four MQ4 sensors have been placed at the top of the physical model in positions from upstream to downstream (two sensors after the upstream coal feeder and the other two sensors located after the downstream feeder), thus the methane concentrations from upstream to downstream of airflow could be detected by the sensor using a data logger and computer. Ultra High Purity (UHP) methane gas was injected into the physical model at 0.1 ; $0.2 ; 0.3 ; 0.4$ and $0.5 \mathrm{~L}$ per minute. Two axial fans (Rayden Fan, $12 \mathrm{~cm} \times 12 \mathrm{~cm} \times 3.8 \mathrm{~cm}$; AC 220/240 V 50/60 Hz; $0.14 \mathrm{~A} ; 0.033-0.055 \mathrm{~m}^{3} / \mathrm{s}$ ) were used at the upstream portal of the physical model, with the purpose of blowing fresh air from outside of the tunnel. Several axial fans with diffuser outlets (Rayden Fan, $9.2 \mathrm{~cm} \times 9.2 \mathrm{~cm} \times 2.5 \mathrm{~cm}$; AC $220 / 240 \mathrm{~V} ; 0.08 \mathrm{~A} ; 0.0245 \mathrm{~m}^{3} / \mathrm{s}$ ) were placed in the physical model to simulate jet fans inside the CRT. A Kestrel 2000 thermo-anemometer (dimension: $122 \mathrm{~mm} \times 42 \mathrm{~mm} \times 20 \mathrm{~mm}$, velocity range of $0.4-40 \mathrm{~m} /$ $\mathrm{s}$, and accuracy of $\pm 0.1 \mathrm{~m} / \mathrm{s}$ ) was used to detect air velocity in the inlet at inside and outlet of the physical model. The air velocity has been measured by using fixedpoint measurement method with 9 (nine) segments on the cross-sectional area of the CRT physical model.

The results of laboratory experiments show that a double-fan-path with straight line fan positions provides better dilution to reduce the concentration of methane gas, in 
Table 2 Parameters of each factor from AHP

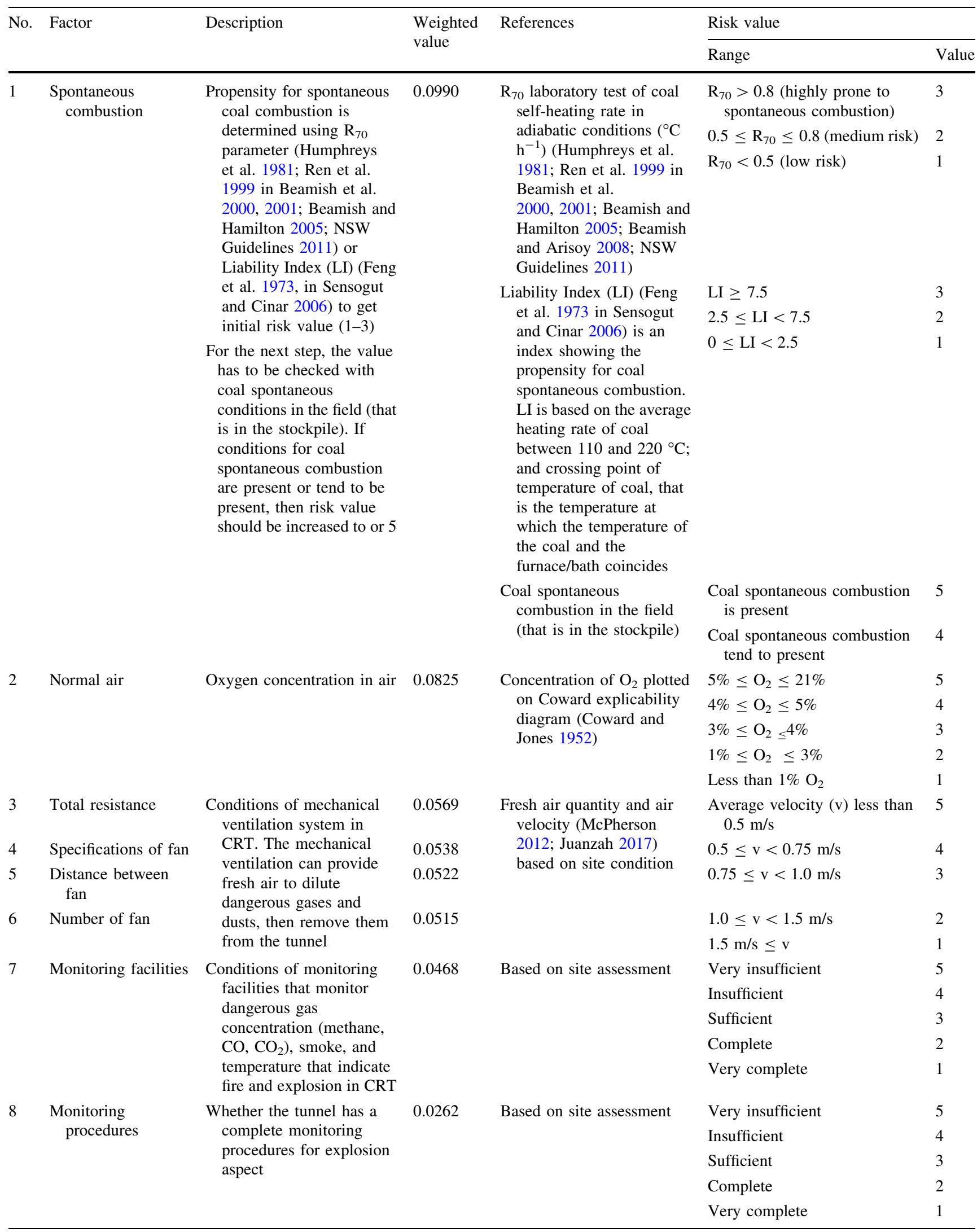


Table 2 continued

\begin{tabular}{|c|c|c|c|c|c|c|}
\hline \multirow[t]{2}{*}{ No. } & \multirow[t]{2}{*}{ Factor } & \multirow[t]{2}{*}{ Description } & \multirow{2}{*}{$\begin{array}{l}\text { Weighted } \\
\text { value }\end{array}$} & \multirow[t]{2}{*}{ References } & \multicolumn{2}{|l|}{ Risk value } \\
\hline & & & & & Range & Value \\
\hline \multirow[t]{5}{*}{9} & \multirow{5}{*}{$\begin{array}{l}\text { Unstable conditions } \\
\text { around the tunnel }\end{array}$} & \multirow{5}{*}{$\begin{array}{l}\text { Explanation of the tunnel } \\
\text { stability conditions }\end{array}$} & \multirow[t]{5}{*}{0.0378} & \multirow[t]{5}{*}{ Based on site assessment } & Very unstable & 5 \\
\hline & & & & & Unstable & 4 \\
\hline & & & & & Stable & 3 \\
\hline & & & & & Stable to very stable & 2 \\
\hline & & & & & Very stable & 1 \\
\hline \multirow[t]{5}{*}{10} & \multirow[t]{5}{*}{ Coal properties } & \multirow{5}{*}{$\begin{array}{l}\text { The influence of coal } \\
\text { properties on methane } \\
\text { content }\end{array}$} & \multirow[t]{5}{*}{0.0365} & \multirow{5}{*}{$\begin{array}{l}\text { The US Bureau of Mines } \\
\text { estimated the methane } \\
\text { content of a coal, which } \\
\text { depends primarily upon } \\
\text { rank and pressure (Kim } \\
\text { 1977) }\end{array}$} & Antracite & 5 \\
\hline & & & & & Low volatile bituminous & 4 \\
\hline & & & & & High volatile bituminous & 3 \\
\hline & & & & & Subbituminous & 2 \\
\hline & & & & & Lignite & 1 \\
\hline \multirow[t]{5}{*}{11} & \multirow{5}{*}{$\begin{array}{l}\text { Coal production } \\
\text { level (related to } \\
\text { methane } \\
\text { concentration in } \\
\text { CRT) }\end{array}$} & \multirow{5}{*}{$\begin{array}{l}\text { The influence of coal } \\
\text { production on methane } \\
\text { concentration }\end{array}$} & \multirow[t]{5}{*}{0.0355} & \multirow{5}{*}{$\begin{array}{l}\text { Fresh air quantity } \\
\text { (McPherson 2012) and } \\
\text { based on site condition }\end{array}$} & More than $30 \mathrm{kton} /$ day & 5 \\
\hline & & & & & $20-30 \mathrm{kton} /$ day & 4 \\
\hline & & & & & $10-20 \mathrm{kton} / \mathrm{day}$ & 3 \\
\hline & & & & & 5-10 kton/day & 2 \\
\hline & & & & & Less than $5 \mathrm{kton} /$ day & 1 \\
\hline \multirow[t]{5}{*}{12} & \multirow{5}{*}{$\begin{array}{l}\text { Time period of coal } \\
\text { in stockpile }\end{array}$} & \multirow{5}{*}{$\begin{array}{l}\text { The influence of the amount } \\
\text { of time the coal is in the } \\
\text { stockpile on spontaneous } \\
\text { combustion and reactivity } \\
\text { of coal }\end{array}$} & \multirow[t]{5}{*}{0.0222} & Heat map of stockpile & More than 3 weeks & 5 \\
\hline & & & & (Pratama 2014; Aristien & 3 weeks & 4 \\
\hline & & & & based on site condition & 2 weeks & 3 \\
\hline & & & & & 1 week & 2 \\
\hline & & & & & Less than 1 day & 1 \\
\hline 13 & Humidity & The influence of humidity & 0.0315 & Based on site assessment, & $\mathrm{RH} \geq 95 \%$ & 5 \\
\hline & & on natural ventilation & & obtained from ventilation & $85 \% \leq \mathrm{RH}<95 \%$ & 4 \\
\hline & & & & Humidity (RH) & $80 \% \leq \mathrm{RH}<85 \%$ & 3 \\
\hline & & & & & $70 \% \leq \mathrm{RH}<80 \%$ & 2 \\
\hline & & & & & $70 \% \leq \mathrm{RH}$ & 1 \\
\hline 14 & Addition of inert & The influence of inert gases & 0.0275 & Based on site assessment, & Not & 5 \\
\hline & & on fire countermeasures & & obtained from ventilation & Has been planned but not ready & 4 \\
\hline & & & & & $\begin{array}{l}\text { Ready to be used but system } \\
\text { has not been developed }\end{array}$ & 3 \\
\hline & & & & & $\begin{array}{l}\text { Ready to be used and system } \\
\text { has been developed }\end{array}$ & 2 \\
\hline & & & & & $\begin{array}{l}\text { Ready to be used and system } \\
\text { has been developed in real } \\
\text { time }\end{array}$ & 1 \\
\hline 15 & Gases & The presence of & 0.0248 & Based on site assessment, & Extremely significant effect & 5 \\
\hline & & combustible gas in the & & obtained from ventilation & Very significant effect & 4 \\
\hline & & & & survey & Significant effect & 3 \\
\hline & & & & & Insignificant effect & 2 \\
\hline & & & & & No effect & 1 \\
\hline 16 & Fire & The presence of fire & 0.0206 & Based on site assessment, & Extremely significant effect & 5 \\
\hline & & potential in the tunnel; & & obtained from ventilation & Very significant effect & 4 \\
\hline & & $\begin{array}{l}\text { how much a nre would } \\
\text { decrease oxygen level in }\end{array}$ & & & Significant effect & 3 \\
\hline & & CRT & & & Insignificant effect & 2 \\
\hline & & & & & No effect & 1 \\
\hline
\end{tabular}


Table 2 continued

\begin{tabular}{|c|c|c|c|c|c|c|}
\hline \multirow[t]{2}{*}{ No. } & \multirow[t]{2}{*}{ Factor } & \multirow[t]{2}{*}{ Description } & \multirow{2}{*}{$\begin{array}{l}\text { Weighted } \\
\text { value }\end{array}$} & \multirow[t]{2}{*}{ References } & \multicolumn{2}{|l|}{ Risk value } \\
\hline & & & & & Range & Value \\
\hline 17 & $\begin{array}{l}\text { Coal production } \\
\text { level (related to } \\
\text { coal dust } \\
\text { concentration in } \\
\text { CRT) }\end{array}$ & $\begin{array}{l}\text { The influence of coal } \\
\text { production on dust } \\
\text { concentration }\end{array}$ & 0.0199 & $\begin{array}{l}\text { Effect of coal production } \\
\text { level on the concentration } \\
\text { of coal dust in the air } \\
\text { (McPherson 2012) }\end{array}$ & $\begin{array}{l}\text { More than } 30 \text { kton/day } \\
20-30 \text { kton/day } \\
10-20 \text { kton/day } \\
5-10 \text { kton/day } \\
\text { Less than } 5 \text { kton/day }\end{array}$ & $\begin{array}{l}5 \\
4 \\
3 \\
2 \\
1\end{array}$ \\
\hline 18 & Design of tunnel & $\begin{array}{l}\text { The influence of tunnel } \\
\text { design on confinement } \\
\text { and dispersion factor }\end{array}$ & 0.0189 & Based on site assessment & $\begin{array}{l}\text { Very disorganized } \\
\text { Disorganized } \\
\text { Fairly organized } \\
\text { Fairly to very organized } \\
\text { Very organized }\end{array}$ & $\begin{array}{l}5 \\
4 \\
3 \\
2 \\
1\end{array}$ \\
\hline 19 & Total moisture & Total moisture of coal & 0.0187 & $\begin{array}{l}\text { Total moisture (TM) affects } \\
\text { on propensity of coal } \\
\text { spontaneous combustion } \\
\text { (Beamish and Hamilton } \\
\text { 2005) and based on site } \\
\text { condition }\end{array}$ & $\begin{array}{l}\mathrm{TM} \leq 2 \% \\
2 \%<\mathrm{TM} \leq 6 \% \\
6 \%<\mathrm{TM} \leq 8 \% \\
8 \%<\mathrm{TM} \leq 10 \% \\
\mathrm{TM}>10 \%\end{array}$ & $\begin{array}{l}5 \\
4 \\
3 \\
2 \\
1\end{array}$ \\
\hline 20 & Cable systems & $\begin{array}{l}\text { Condition of cable systems } \\
\text { in the tunnel, as a } \\
\text { potential source of } \\
\text { ignition }\end{array}$ & 0.0182 & Based on site assessment & $\begin{array}{l}\text { Very disorganized } \\
\text { Disorganized } \\
\text { Fairly organized } \\
\text { Fairly to very organized } \\
\text { Very organized }\end{array}$ & $\begin{array}{l}5 \\
4 \\
3 \\
2 \\
1\end{array}$ \\
\hline 21 & Size of void & $\begin{array}{l}\text { The effect of void size on } \\
\text { methane trapping }\end{array}$ & 0.0174 & $\begin{array}{l}\text { Size of void affect to } \\
\text { methane distribution in } \\
\text { tunnel (Pratama 2016; } \\
\text { Kusuma 2016b; Juanzah } \\
\text { 2017) and based on site } \\
\text { condition }\end{array}$ & $\begin{array}{l}\text { Extremely significant effect } \\
\text { Very significant effect } \\
\text { Significant effect } \\
\text { Insignificant effect } \\
\text { No effect }\end{array}$ & $\begin{array}{l}5 \\
4 \\
3 \\
2 \\
1\end{array}$ \\
\hline 22 & Friction & $\begin{array}{l}\text { Sparks by friction, as a } \\
\text { potential of heat source } \\
\text { for explosion }\end{array}$ & 0.0165 & Based on site assessment & $\begin{array}{l}\text { Very often } \\
\text { Often } \\
\text { Occasionally } \\
\text { Rarely } \\
\text { Very rarely }\end{array}$ & $\begin{array}{l}5 \\
4 \\
3 \\
2 \\
1\end{array}$ \\
\hline 23 & Monitors layout & $\begin{array}{l}\text { Whether the tunnels has a } \\
\text { good monitor layout }\end{array}$ & 0.0139 & Based on site assessment & $\begin{array}{l}\text { Very insufficient } \\
\text { Insufficient } \\
\text { Sufficient } \\
\text { Good } \\
\text { Very good }\end{array}$ & $\begin{array}{l}5 \\
4 \\
3 \\
2 \\
1\end{array}$ \\
\hline 24 & Monitoring staff & $\begin{array}{l}\text { Whether adequate personnel } \\
\text { are available to monitor } \\
\text { the system }\end{array}$ & 0.0131 & Based on site assessment & $\begin{array}{l}\text { Very inadequate } \\
\text { Inadequate } \\
\text { Adequate } \\
\text { Adequate to very adequate } \\
\text { Very adequate }\end{array}$ & $\begin{array}{l}5 \\
4 \\
3 \\
2 \\
1\end{array}$ \\
\hline
\end{tabular}


Table 2 continued

\begin{tabular}{|c|c|c|c|c|c|c|}
\hline \multirow[t]{2}{*}{ No. } & \multirow[t]{2}{*}{ Factor } & \multirow[t]{2}{*}{ Description } & \multirow{2}{*}{$\begin{array}{l}\text { Weighted } \\
\text { value }\end{array}$} & \multirow[t]{2}{*}{ References } & \multicolumn{2}{|l|}{ Risk value } \\
\hline & & & & & Range & Value \\
\hline \multirow[t]{5}{*}{25} & \multirow[t]{5}{*}{ Volatile matter } & \multirow[t]{5}{*}{ Volatile matter of coal } & \multirow[t]{5}{*}{0.0128} & \multirow{5}{*}{$\begin{array}{l}\text { According to Uludag (2007) } \\
\text { in Nalbandian (2010), it is } \\
\text { generally agreed that } \\
\text { spontaneous combustion } \\
\text { is a rank-related } \\
\text { phenomenon. As Volatile } \\
\text { Matter (VM) and Oxygen } \\
\text { content increase } \\
\text { (indicative of decrease in } \\
\text { rank), the rate of self- } \\
\text { heating is also raised }\end{array}$} & $\begin{array}{l}\mathrm{VM} \text { has very high contribution } \\
\text { to } \mathrm{R}_{70} \text { self-heating rate }\end{array}$ & 5 \\
\hline & & & & & $\begin{array}{l}\mathrm{VM} \text { has high contribution to } \\
\mathrm{R}_{70} \text { self-heating rate }\end{array}$ & 4 \\
\hline & & & & & $\begin{array}{l}\mathrm{VM} \text { has contribution to } \mathrm{R}_{70} \\
\text { self-heating rate }\end{array}$ & 3 \\
\hline & & & & & $\begin{array}{l}\text { VM has less contribution to } \\
\mathrm{R}_{70} \text { self-heating rate }\end{array}$ & 2 \\
\hline & & & & & $\begin{array}{l}\text { VM has no contribution to } R_{70} \\
\text { self-heating rate }\end{array}$ & 1 \\
\hline \multirow[t]{5}{*}{26} & \multirow[t]{5}{*}{ Fireproof } & \multirow{5}{*}{$\begin{array}{l}\text { Is fireproof material present } \\
\text { in the tunnels to avoid } \\
\text { spreading of fire through } \\
\text { the materials }\end{array}$} & \multirow[t]{5}{*}{0.0128} & \multirow{5}{*}{$\begin{array}{l}\text { In general, three types of } \\
\text { materials are used for } \\
\text { mine conveyor belts, } \\
\text { namely, styrene- } \\
\text { butadiene rubber, } \\
\text { neoprene, and } \\
\text { polyvinylchloride } \\
\text { (McPherson 2012) }\end{array}$} & Very high risk & 5 \\
\hline & & & & & High risk & 4 \\
\hline & & & & & Medium risk & 3 \\
\hline & & & & & Low risk & 2 \\
\hline & & & & & Very low risk & 1 \\
\hline \multirow[t]{5}{*}{27} & \multirow[t]{5}{*}{ Heat } & \multirow{5}{*}{$\begin{array}{l}\text { How the presence of heat } \\
\text { condition (an ignition) in } \\
\text { the tunnel }\end{array}$} & \multirow[t]{5}{*}{0.0124} & \multirow{5}{*}{$\begin{array}{l}\text { Heat contributed to ignition: } \\
\text { air temperature, heat from } \\
\text { increasing of temperature } \\
\text { on equipment surfaces } \\
\text { (Iqbal 2016; Kusuma } \\
\text { 2016a) and based on site } \\
\text { condition }\end{array}$} & Very high risk & 5 \\
\hline & & & & & High risk & 4 \\
\hline & & & & & Medium risk & 3 \\
\hline & & & & & Low risk & 2 \\
\hline & & & & & Very low risk & 1 \\
\hline \multirow[t]{5}{*}{28} & \multirow[t]{5}{*}{ Number of void } & \multirow{5}{*}{$\begin{array}{l}\text { Whether there is a void that } \\
\text { became a methane } \\
\text { trapping }\end{array}$} & \multirow[t]{5}{*}{0.0096} & \multirow{5}{*}{$\begin{array}{l}\text { Methane distribution in } \\
\text { tunnel (Pratama 2016; } \\
\text { Kusuma 2016b; Juanzah } \\
\text { 2017) and based on site } \\
\text { condition }\end{array}$} & Very high risk & 5 \\
\hline & & & & & High risk & 4 \\
\hline & & & & & Medium risk & 3 \\
\hline & & & & & Low risk & 2 \\
\hline & & & & & Very low risk & 1 \\
\hline \multirow[t]{5}{*}{29} & Electric motors on & Conveyor belt can produce & 0.0091 & Based on site assessment & Very often & 5 \\
\hline & & sparks from its electrical & & & Often & 4 \\
\hline & & & & & Occasionally & 3 \\
\hline & & & & & Rarely & 2 \\
\hline & & & & & Very rarely & 1 \\
\hline 30 & Dust particle & How the effects of relative & 0.0089 & Based on site assessment & Very high risk & 5 \\
\hline & coagulation due to & humidity on the & & & High risk & 4 \\
\hline & & & & & Medium risk & 3 \\
\hline & & & & & Low risk & 2 \\
\hline & & & & & Very low risk & 1 \\
\hline 31 & Monitoring tools & The tunnel have a complete & 0.0069 & Based on site assessment & Very less & 5 \\
\hline & & monitoring Tools & & & Less & 4 \\
\hline & & & & & Sufficient & 3 \\
\hline & & & & & Complete & 2 \\
\hline & & & & & Very complete & 1 \\
\hline
\end{tabular}


Table 2 continued

\begin{tabular}{|c|c|c|c|c|c|c|}
\hline \multirow[t]{2}{*}{ No. } & \multirow[t]{2}{*}{ Factor } & \multirow[t]{2}{*}{ Description } & \multirow{2}{*}{$\begin{array}{l}\text { Weighted } \\
\text { value }\end{array}$} & \multirow[t]{2}{*}{ References } & \multicolumn{2}{|l|}{ Risk value } \\
\hline & & & & & Range & Value \\
\hline \multirow[t]{5}{*}{32} & \multirow[t]{5}{*}{ Combustion } & \multirow{5}{*}{$\begin{array}{l}\text { Potential for combustion in } \\
\text { the tunnel }\end{array}$} & \multirow[t]{5}{*}{0.0069} & \multirow[t]{5}{*}{ Based on site assessment } & Combustion exist & 5 \\
\hline & & & & & $\begin{array}{l}\text { Combustion has a potential to } \\
\text { exist }\end{array}$ & 4 \\
\hline & & & & & $\begin{array}{l}\text { Combustion has a potential to } \\
\text { exist when triggered by other } \\
\text { factor }\end{array}$ & 3 \\
\hline & & & & & $\begin{array}{l}\text { Very small combustion } \\
\text { potential }\end{array}$ & 2 \\
\hline & & & & & Combustion does not exist & 1 \\
\hline \multirow[t]{5}{*}{33} & \multirow[t]{5}{*}{ Ash content } & \multirow[t]{5}{*}{ Ash content of coal } & \multirow[t]{5}{*}{0.0064} & \multirow{5}{*}{$\begin{array}{l}\text { Ash content effects on } \\
\text { propensity of coal } \\
\text { spontaneous combustion } \\
\text { (Beamish and Hamilton } \\
\text { 2005) }\end{array}$} & $\begin{array}{l}\text { Sub-bituminous: ash } \\
\qquad(\mathrm{db}) \leq 5 \%\end{array}$ & 5 \\
\hline & & & & & $\begin{array}{l}\text { Sub-bituminous: } 5 \%<\text { Ash } \\
\qquad(\mathrm{db}) \leq 30 \%\end{array}$ & 4 \\
\hline & & & & & $\begin{array}{l}\text { Sub-bituminous: } 30 \%<\text { Ash } \\
\quad(\mathrm{db}) \leq 40 \%\end{array}$ & 3 \\
\hline & & & & & $\begin{array}{l}\text { Medium-high volatile } \\
\text { bituminous: } 5 \%<\text { ash } \\
\text { (db) } \leq 25 \%\end{array}$ & 2 \\
\hline & & & & & $\begin{array}{l}\text { Medium-high volatile } \\
\quad \text { bituminous: } \text { ash }(\mathrm{db}) \geq 25 \%\end{array}$ & 1 \\
\hline \multirow[t]{5}{*}{34} & \multirow{5}{*}{$\begin{array}{l}\text { Coal size in } \\
\text { stockpile }\end{array}$} & \multirow{5}{*}{$\begin{array}{l}\text { The influence of the fineness } \\
\text { of the coal in the } \\
\text { stockpile }\end{array}$} & \multirow[t]{5}{*}{0.0061} & \multirow[t]{5}{*}{ Based on site assessment } & Very high risk & 5 \\
\hline & & & & & High risk & 4 \\
\hline & & & & & Medium risk & 3 \\
\hline & & & & & Low risk & 2 \\
\hline & & & & & Very low risk & 1 \\
\hline \multirow[t]{5}{*}{35} & \multirow{5}{*}{$\begin{array}{l}\text { Ambient } \\
\text { temperature }\end{array}$} & \multirow{5}{*}{$\begin{array}{l}\text { The influence of ambient } \\
\text { temperature on natural } \\
\text { ventilation }\end{array}$} & \multirow[t]{5}{*}{0.0050} & \multirow{5}{*}{$\begin{array}{l}\text { Based on site assessment, } \\
\text { obtained from ventilation } \\
\text { survey of dry bulb } \\
\text { temperature }\left(\mathrm{T}_{\mathrm{d}}\right)\end{array}$} & $\mathrm{T}_{\mathrm{d}} \geq 33^{\circ} \mathrm{C}$ & 5 \\
\hline & & & & & $30{ }^{\circ} \mathrm{C} \leq \mathrm{T}_{\mathrm{d}}<33{ }^{\circ} \mathrm{C}$ & 4 \\
\hline & & & & & $27^{\circ} \mathrm{C} \leq \mathrm{T}_{\mathrm{d}}<30^{\circ} \mathrm{C}$ & 3 \\
\hline & & & & & $24{ }^{\circ} \mathrm{C} \leq \mathrm{T}_{\mathrm{d}}<27^{\circ} \mathrm{C}$ & 2 \\
\hline & & & & & $24^{\circ} \mathrm{C} \leq \mathrm{T}_{\mathrm{d}}$ & 1 \\
\hline \multirow[t]{5}{*}{36} & Electric motors in & Sparks from the fan electric & 0.0049 & Based on site assessment & Very high risk & 5 \\
\hline & & motors are a potential & & & High risk & 4 \\
\hline & & & & & Medium risk & 3 \\
\hline & & & & & Low risk & 2 \\
\hline & & & & & Very low risk & 1 \\
\hline 37 & Flame temperature & The influence of flame & 0.0047 & Based on site assessment & No effect & 5 \\
\hline & level & temperature level on the & & & Insignificant effect & 4 \\
\hline & & combustible gas & & & Significant effect & 3 \\
\hline & & & & & Very significant effect & 2 \\
\hline & & & & & Extremely significant effect & 1 \\
\hline 38 & Time to extinction & The influence of time to & 0.0047 & Based on site assessment & Extremely significant effect & 5 \\
\hline & & extinction of fire on the & & & Very significant effect & 4 \\
\hline & & combustible gas (such as & & & Significant effect & 3 \\
\hline & & $\mathrm{CO})$ & & & Insignificant effect & 2 \\
\hline & & & & & No effect & 1 \\
\hline
\end{tabular}


Table 2 continued

\begin{tabular}{|c|c|c|c|c|c|c|}
\hline \multirow[t]{2}{*}{ No. } & \multirow[t]{2}{*}{ Factor } & \multirow[t]{2}{*}{ Description } & \multirow{2}{*}{$\begin{array}{l}\text { Weighted } \\
\text { value }\end{array}$} & \multirow[t]{2}{*}{ References } & \multicolumn{2}{|l|}{ Risk value } \\
\hline & & & & & Range & Value \\
\hline \multirow[t]{5}{*}{39} & \multirow{5}{*}{$\begin{array}{l}\text { Type of agent (used } \\
\text { in fire } \\
\text { extinguishment) }\end{array}$} & \multirow{5}{*}{$\begin{array}{l}\text { The influence of type of } \\
\text { agent on the emergence of } \\
\text { flammable gas }\end{array}$} & \multirow[t]{5}{*}{0.0047} & \multirow[t]{5}{*}{ Based on site assessment } & Extremely significant effect & 5 \\
\hline & & & & & Very significant effect & 4 \\
\hline & & & & & Significant effect & 3 \\
\hline & & & & & Insignificant effect & 2 \\
\hline & & & & & No effect & 1 \\
\hline \multirow[t]{5}{*}{40} & \multirow{5}{*}{$\begin{array}{l}\text { Existence of winds } \\
\text { (Wind speed, WS) }\end{array}$} & \multirow{5}{*}{$\begin{array}{l}\text { The influence of winds on } \\
\text { natural ventilation }\end{array}$} & \multirow[t]{5}{*}{0.0047} & \multirow{5}{*}{$\begin{array}{l}\text { Based on site assessment, } \\
\text { obtained from ventilation } \\
\text { survey }\end{array}$} & $\mathrm{WS} \leq 0.2 \mathrm{~m} / \mathrm{s}$ & 5 \\
\hline & & & & & $0.2 \mathrm{~m} / \mathrm{s}<\mathrm{WS} \leq 1.5 \mathrm{~m} / \mathrm{s}$ & 4 \\
\hline & & & & & $1.5 \mathrm{~m} / \mathrm{s}<\mathrm{WS} \leq 3.3 \mathrm{~m} / \mathrm{s}$ & 3 \\
\hline & & & & & $3.3 \mathrm{~m} / \mathrm{s}<\mathrm{WS} \leq 5.4 \mathrm{~m} / \mathrm{s}$ & 2 \\
\hline & & & & & $5.4 \mathrm{~m} / \mathrm{s}<\mathrm{WS} \leq 10 \mathrm{~m} / \mathrm{s}$ & 1 \\
\hline \multirow[t]{5}{*}{41} & \multirow[t]{5}{*}{$\begin{array}{l}\text { Barometric pressure } \\
\text { (BP) }\end{array}$} & \multirow{5}{*}{$\begin{array}{l}\text { The influence of barometric } \\
\text { pressure on natural } \\
\text { ventilation }\end{array}$} & \multirow[t]{5}{*}{0.0045} & \multirow{5}{*}{$\begin{array}{l}\text { Based on site assessment, } \\
\text { obtained from ventilation } \\
\text { survey }\end{array}$} & $\begin{array}{l}\text { BP difference between inlet } \\
\text { and outlet about } 0 \mathrm{~Pa}\end{array}$ & 5 \\
\hline & & & & & $0 \mathrm{~Pa}<\mathrm{BP} \leq 5 \mathrm{~Pa}$ & 4 \\
\hline & & & & & $5 \mathrm{~Pa}<\mathrm{BP} \leq 15 \mathrm{~Pa}$ & 3 \\
\hline & & & & & $15 \mathrm{~Pa}<\mathrm{BP} \leq 25 \mathrm{~Pa}$ & 2 \\
\hline & & & & & $\begin{array}{l}\text { BP difference between inlet } \\
\text { and outlet about } 25 \mathrm{~Pa}\end{array}$ & 1 \\
\hline \multirow[t]{5}{*}{42} & \multirow[t]{5}{*}{ Pressure } & \multirow{5}{*}{$\begin{array}{l}\text { Sparks can come from and } \\
\text { are a potential heat source } \\
\text { for explosion }\end{array}$} & \multirow[t]{5}{*}{0.0041} & Based on site assessment & Very often & 5 \\
\hline & & & & & Often & 4 \\
\hline & & & & & Occasionally & 3 \\
\hline & & & & & Rarely & 2 \\
\hline & & & & & Very Rarely & 1 \\
\hline 43 & Sulphur & Sulphur content of coal & 0.0038 & $\begin{array}{l}\text { Based on site assessment, } \\
\text { Nalbandian }(2010)\end{array}$ & $\begin{array}{l}\text { Total sulphur }(\mathrm{TS})>2 \% \\
\quad \text { (dominated by pyritic } \\
\text { sulphur) }\end{array}$ & 5 \\
\hline & & & & & $1.5 \%<\mathrm{TS} \leq 2 \%$ & 4 \\
\hline & & & & & $1.0 \%<\mathrm{TS} \leq 1.5 \%$ & 3 \\
\hline & & & & & $0.1 \%<\mathrm{TS} \leq 1 \%$ & 2 \\
\hline & & & & & $\mathrm{TS} \leq 0.1 \%$ & 1 \\
\hline 44 & Static electricity & Sparks can come from static & 0.0032 & Based on site assessment & Very often & 5 \\
\hline & & electricity and are a & & & Often & 4 \\
\hline & & explosion & & & Occasionally & 3 \\
\hline & & & & & Rarely & 2 \\
\hline & & & & & Very rarely & 1 \\
\hline 45 & Position of void & The effect of void position & 0.0030 & Methane distribution in & Extremely significant effect & 5 \\
\hline & & in the CRT on methane & & tunnels (Pratama 2016; & Very significant effect & 4 \\
\hline & & & & $\begin{array}{l}\text { Kusuma } 2010 b \text {; Juanzan } \\
\text { 2017) based on site }\end{array}$ & Significant effect & 3 \\
\hline & & & & condition & Insiginificant effect & 2 \\
\hline & & & & & No effect & 1 \\
\hline 46 & Exhaust fan & Whether the exhaust fan are & 0.0022 & Based on site assessment & Not functioning & 5 \\
\hline & & properly functioning to & & & Few functioning & 4 \\
\hline & & & & & Sufficient functioning & 3 \\
\hline & & & & & Sufficient—fully functioning & 2 \\
\hline & & & & & Fully functioning & 1 \\
\hline
\end{tabular}


Table 2 continued

\begin{tabular}{|c|c|c|c|c|c|c|}
\hline \multirow[t]{2}{*}{ No. } & \multirow[t]{2}{*}{ Factor } & \multirow[t]{2}{*}{ Description } & \multirow{2}{*}{$\begin{array}{l}\text { Weighted } \\
\text { value }\end{array}$} & \multirow[t]{2}{*}{ References } & \multicolumn{2}{|l|}{ Risk value } \\
\hline & & & & & Range & Value \\
\hline \multirow[t]{5}{*}{47} & \multirow[t]{5}{*}{ Electronic devices } & \multirow{5}{*}{$\begin{array}{l}\text { Incendiary sparks produced } \\
\text { by electronic devices }\end{array}$} & \multirow[t]{5}{*}{0.0021} & \multirow[t]{5}{*}{ Based on site assessment } & Very often & 5 \\
\hline & & & & & Often & 4 \\
\hline & & & & & Occasionally & 3 \\
\hline & & & & & Rarely & 2 \\
\hline & & & & & Very rarely & 1 \\
\hline \multirow[t]{5}{*}{48} & \multirow{5}{*}{$\begin{array}{l}\text { Type of fluid (used } \\
\text { in dust spraying) }\end{array}$} & \multirow{5}{*}{$\begin{array}{l}\text { The effects of type of fluid } \\
\text { used for dust spraying }\end{array}$} & \multirow[t]{5}{*}{0.0017} & \multirow[t]{5}{*}{ Based on site assessment } & Extremely significant effect & 5 \\
\hline & & & & & Very significant effect & 4 \\
\hline & & & & & Significant effect & 3 \\
\hline & & & & & Insignificant effect & 2 \\
\hline & & & & & No effect & 1 \\
\hline \multirow[t]{5}{*}{49} & \multirow[t]{5}{*}{ Non fireproof } & \multirow{5}{*}{$\begin{array}{l}\text { The effect of non-fireproof } \\
\text { composition on } \\
\text { flammable gases } \\
\text { produced }\end{array}$} & \multirow[t]{5}{*}{0.0014} & \multirow{5}{*}{$\begin{array}{l}\text { In general, three types of } \\
\text { materials are used for } \\
\text { mine conveyor belts, } \\
\text { namely, styrene- } \\
\text { butadiene rubber (SBR), } \\
\text { neoprene (NP) and } \\
\text { polyvinylchloride (PVC) } \\
\text { (McPherson 2012) }\end{array}$} & Extremely significant effect & 5 \\
\hline & & & & & Very significant effect & 4 \\
\hline & & & & & Significant effect & 3 \\
\hline & & & & & Insignificant effect & 2 \\
\hline & & & & & No effect & 1 \\
\hline \multirow[t]{5}{*}{50} & \multirow[t]{5}{*}{ Spraying pressure } & \multirow{5}{*}{$\begin{array}{l}\text { The effects of spraying } \\
\text { pressure on dust spraying }\end{array}$} & \multirow[t]{5}{*}{0.0004} & \multirow[t]{5}{*}{ Based on site assessment } & Extremely significant effect & 5 \\
\hline & & & & & Very significant effect & 4 \\
\hline & & & & & Significant effect & 3 \\
\hline & & & & & Insignificant effect & 2 \\
\hline & & & & & No effect & 1 \\
\hline \multirow[t]{5}{*}{51} & \multirow[t]{5}{*}{ Type of nozzle } & \multirow{5}{*}{$\begin{array}{l}\text { The effects of type of nozzle } \\
\text { on dust spraying }\end{array}$} & \multirow[t]{5}{*}{0.0002} & Based on site assessment & Extremely significant effect & 5 \\
\hline & & & & & Very significant effect & 4 \\
\hline & & & & & Significant effect & 3 \\
\hline & & & & & Insignificant effect & 2 \\
\hline & & & & & No effect & 1 \\
\hline 52 & Diffusion coefficient & $\begin{array}{l}\text { The effectiveness of dilution } \\
\text { of methane }\end{array}$ & 0.0046 & $\begin{array}{l}\text { Methane gas diffusion } \\
\text { coefficient related with } \\
\text { ventilation condition in }\end{array}$ & $\begin{array}{l}\text { Very small diffusion } \\
\text { coefficient (dilution is very } \\
\text { ineffective) }\end{array}$ & 5 \\
\hline & & & & $\begin{array}{l}\text { CRT (Juanzah 2017) and } \\
\text { based on site condition }\end{array}$ & $\begin{array}{l}\text { Small diffusion coefficient } \\
\text { (dilution is ineffective) }\end{array}$ & 4 \\
\hline & & & & & $\begin{array}{l}\text { Enough diffusion coefficient } \\
\text { (dilution is good) }\end{array}$ & 3 \\
\hline & & & & & $\begin{array}{l}\text { High diffusion coefficient } \\
\text { (dilution is effective) }\end{array}$ & 2 \\
\hline & & & & & $\begin{array}{l}\text { Ideal diffusion coefficient } \\
\text { (dilution is very effective) }\end{array}$ & 1 \\
\hline
\end{tabular}

Table 3 CRT explosion risk classification based on total risk rating

\begin{tabular}{lll}
\hline Class & Total risk rating & Risk classification \\
\hline 1 & $0 \leq \mathrm{TRR}<100$ & Very low \\
2 & $100 \leq \mathrm{TRR}<200$ & Low \\
3 & $200 \leq \mathrm{TRR}<300$ & Medium \\
4 & $300 \leq \mathrm{TRR}<400$ & High \\
5 & TRR $\geq 400$ & Very high \\
\hline
\end{tabular}

comparison to the double-fan-path with zigzag fan positions, and single-fan-path configuration. This is shown by the average air velocity measured inside and in the outlet of the tunnel: $0.70 \mathrm{~m} / \mathrm{s}$ for a single-fan-path; $0.77 \mathrm{~m} / \mathrm{s}$ for a double-fan-path with zigzag fan positions; and $1.01 \mathrm{~m} / \mathrm{s}$ for a double-fan-path with straight line fan positions, as shown in Fig. 7. Relatively higher air velocity is more effective at reducing the methane gas concentration inside the CRT than lower air velocity. 


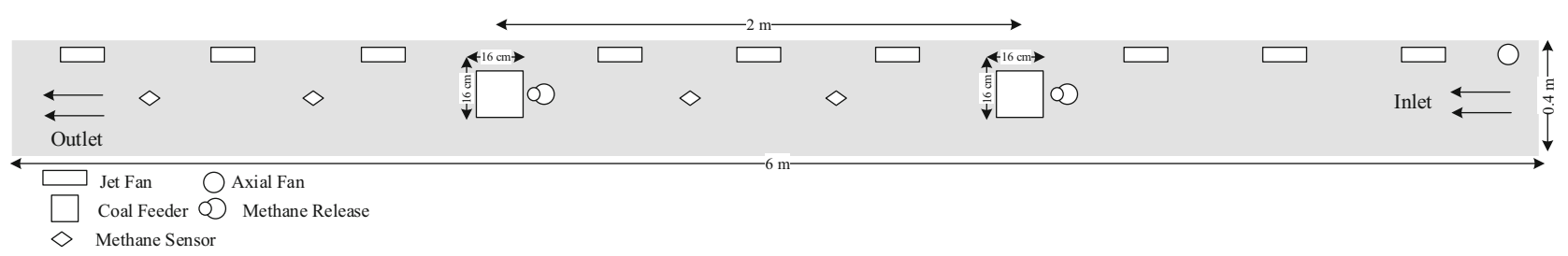

(a)

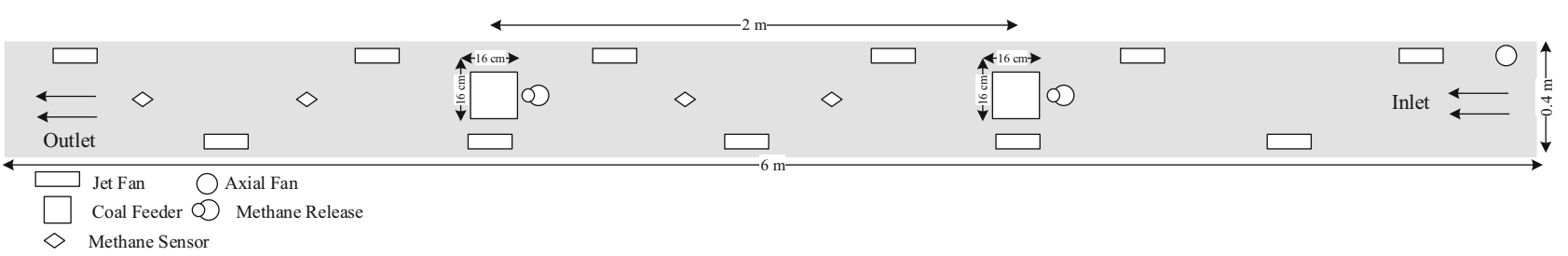

(b)

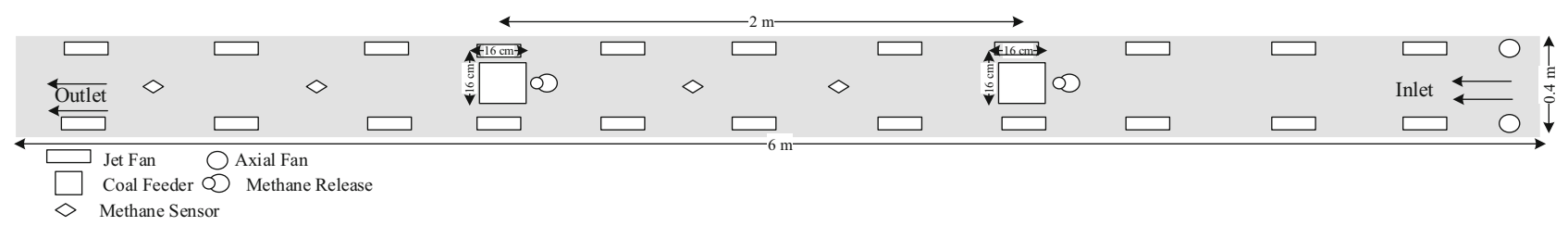

(c)

Fig. 5 Schematic side view of CRT physical model with differences in jet fan configurations. a Single-fan-path configurations (plan view). b Double-fan-path with zigzag fan positions (plan view). c Double-fan-path with straight line fan positions (plan view)

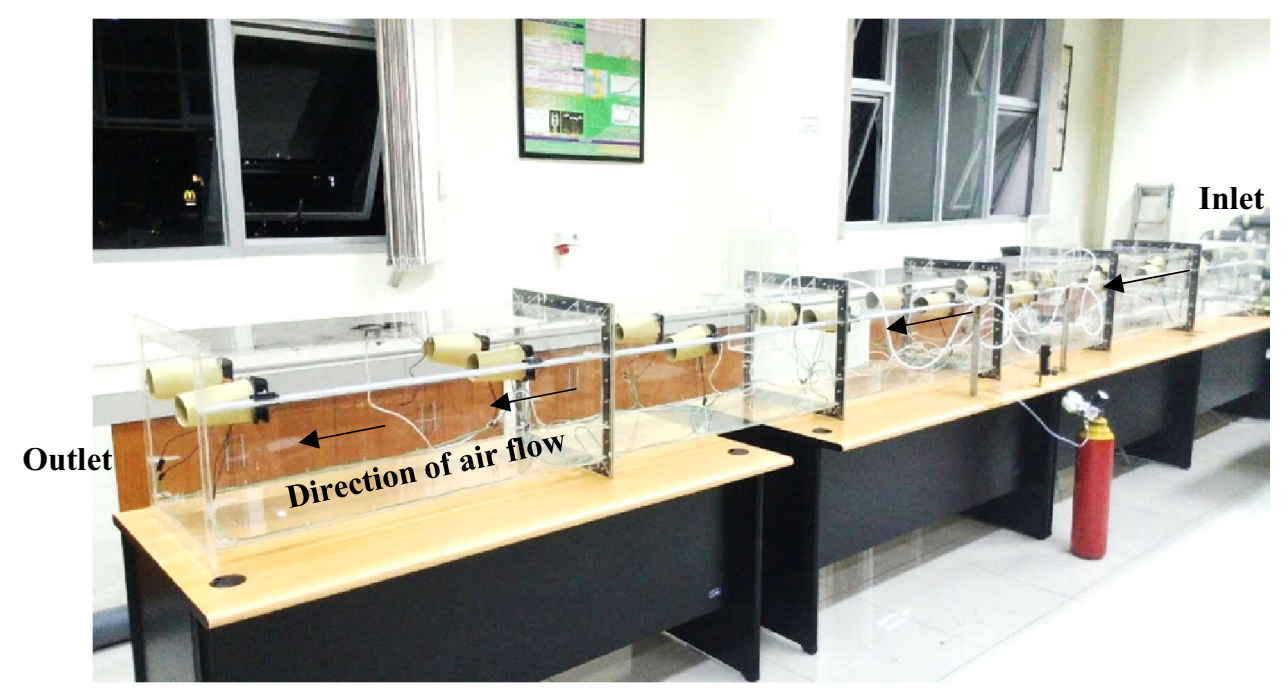

Fig. 6 Laboratory physical model of CRT

The indication of methane dilution in CRT was represented by the effective dispersion coefficient $(E)$, that the bigger dispersion coefficient, the lower concentration of methane gas at the outlet, and vice versa. The methane dispersion coefficients in the CRT laboratory physical model which were estimated by concentration-time matching curves: $0.078-0.089 \mathrm{~m}^{2} / \mathrm{s}$ for a single-fan-path,
0.089-0.094 $\mathrm{m}^{2} / \mathrm{s}$ for a double-fan-path with zigzag fan, and $0.110-0.122 \mathrm{~m}^{2} / \mathrm{s}$ for a double-fan-path with straight line fan positions (details are shown in Table 4). These result are in good agreement with the field measurement assessment results, that were represented indirectly by fine coal dust concentrations assessed in real CRT conditions. 


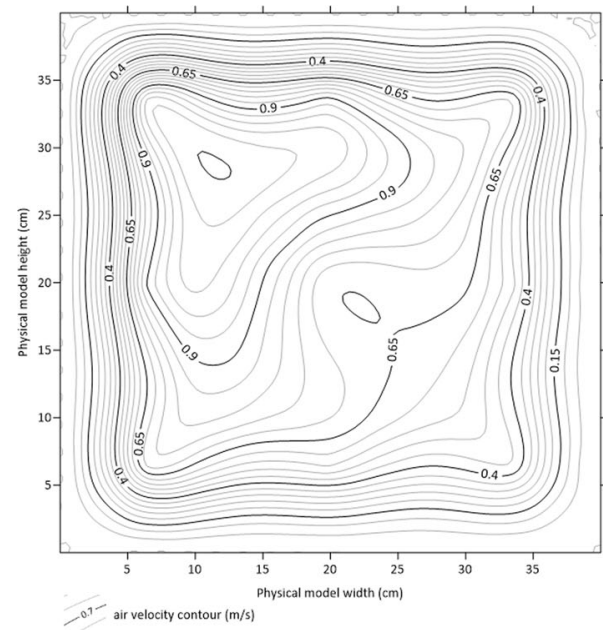

(a)

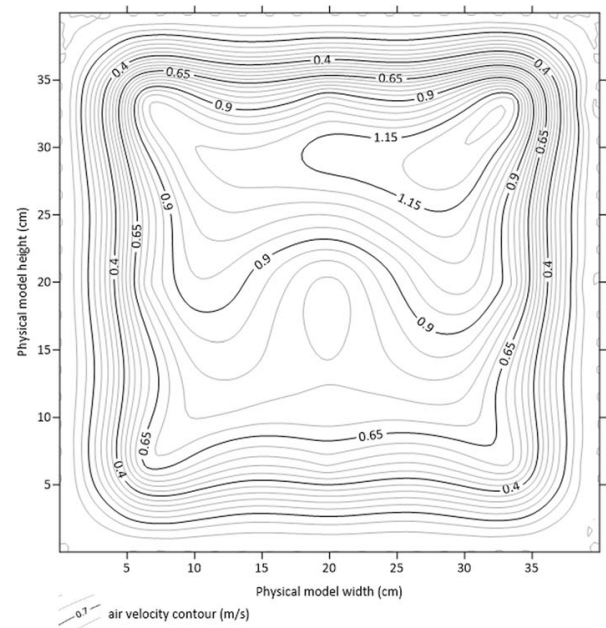

(b)

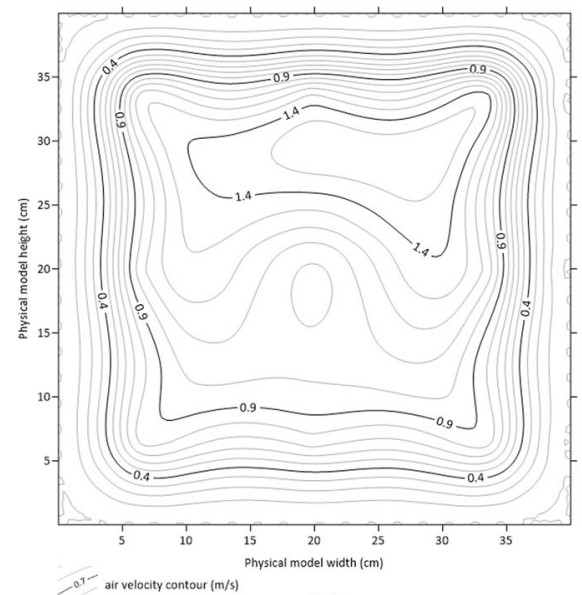

(c)

Fig. 7 Average air velocity measured in the CRT physical model. a Single-fan-path configurations. b Double-fan-path with zigzag fan positions. c Double-fan-path with straight line fan positions
Table 4 Dispersion coefficient $(E)$ of methane gas-air in CRT Physical model

\begin{tabular}{llllll}
\hline Jet fan & \multicolumn{6}{l}{ Dispersion coefficient, $E\left(\mathrm{~m}^{2} / \mathrm{s}\right)$} \\
\cline { 2 - 6 } & 0.1 & 0.2 & 0.3 & 0.4 & 0.5 \\
& $\mathrm{~L} / \mathrm{min}$ & $\mathrm{L} / \mathrm{min}$ & $\mathrm{L} / \mathrm{min}$ & $\mathrm{L} / \mathrm{min}$ & $\mathrm{L} / \mathrm{min}$ \\
\hline Single & 0.087 & 0.078 & 0.089 & 0.087 & 0.087 \\
Zig-zag & 0.094 & 0.089 & 0.091 & 0.092 & 0.089 \\
Double & 0.120 & 0.122 & 0.110 & 0.119 & 0.118 \\
\hline
\end{tabular}

\section{Case study}

To apply the risk assessment methods that have been developed, assessments were conducted in two CRTs in Indonesia, namely the CRT in Mine A and the CRT in Mine B. The type of coal and CRT dimensions are similar between these two CRTs. However, the ventilation conditions and coal stockpile conditions (stockpile height and storage time) are different; specifically, Mine A has more unfavorable conditions related to explosion risk than Mine B.

Significant data have been collected and various measurements have been carried out to assess the explosion risk of CRT. Field measurement activities were conducted: temperature measurements on the coal stockpiles to evaluate the coal spontaneous combustion factor, measurement of coal dust concentration and air velocity inside the CRT tunnels to evaluate the effectiveness of ventilation system for reducing coal dust concentration in the tunnels (Figs. 8, 9 and 10). The measurements of temperature on the coal stockpile were conducted using APPA 51 device, with $\mathrm{K}$-type thermocouple (measurement range of -50 to $1300{ }^{\circ} \mathrm{C}$ with resolution of $0.1{ }^{\circ} \mathrm{C}$ ). Coal stockpile has

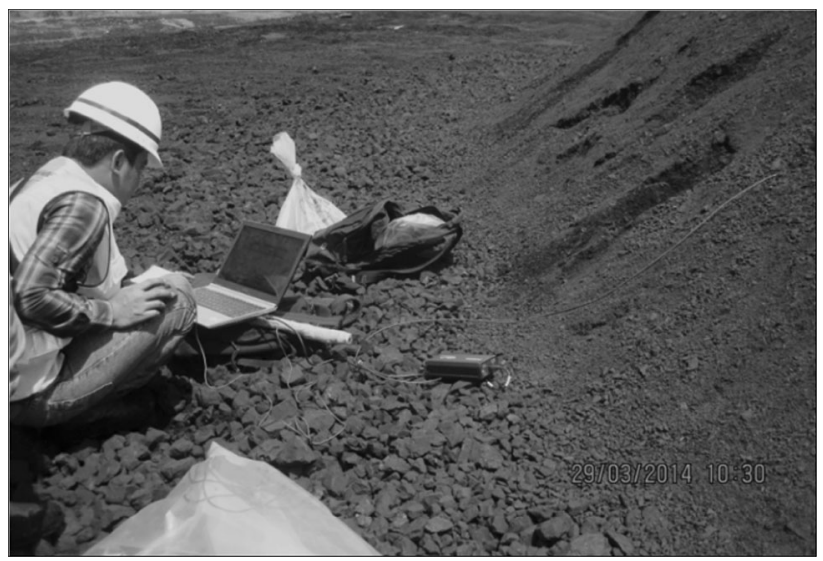

Fig. 8 Temperature measurement on coal stockpile located above the CRT 


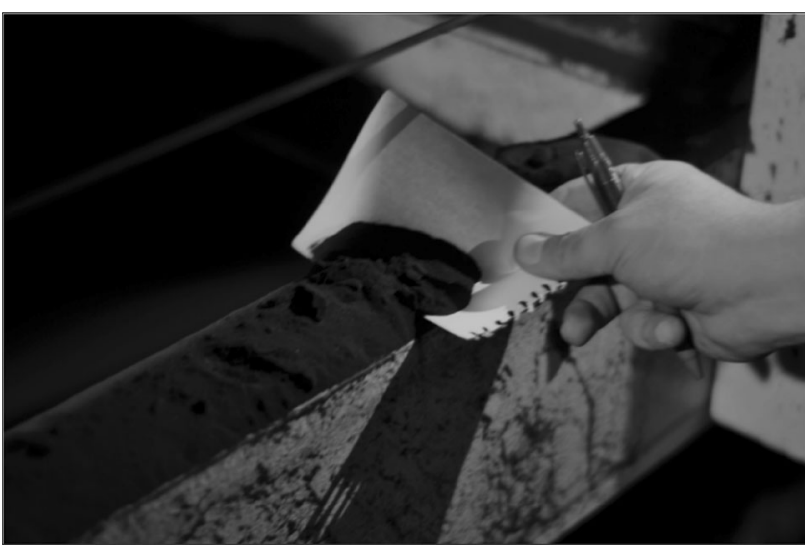

Fig. 9 Dust sampling inside the CRT

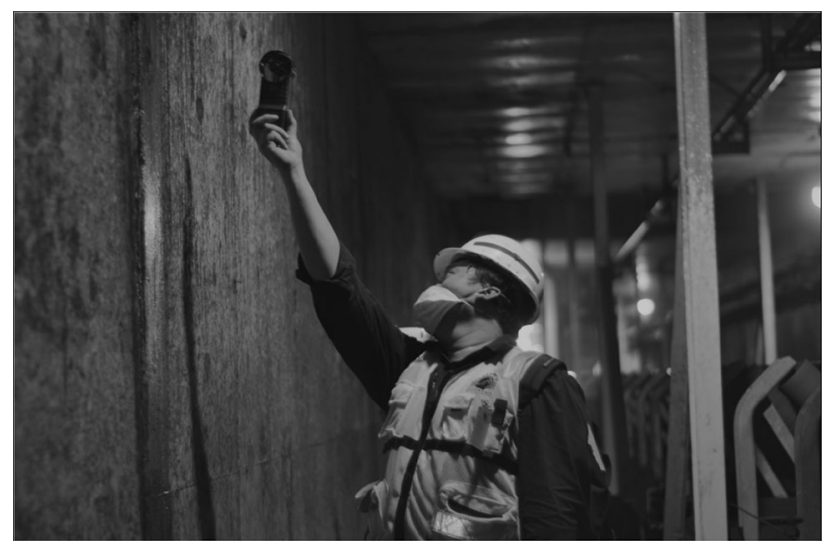

Fig. 10 Air velocity measurement inside the CRT

variation of The measurements of air velocity were conducted using vane anemometer (Dwyer 8904 Rotary Vane Thermo- Anemometer, velocity range of $0.4-30 \mathrm{~m} / \mathrm{s}$, and accuracy of $\pm 0.2 \mathrm{~m} / \mathrm{s}$ ). The coal dust conditions were estimated by visual observation. The field measurement results are described in Table 5.

\subsection{Explosion risk assessment of CRT in Mine $A$ and Mine B using developed AHP method}

The CRT in Mine A is $250 \mathrm{~m}$ in length and $4 \mathrm{~m}$ in width and height. In this CRT, there are some equipment such as a conveyor, coal valve, fan, and deluge system. Three jet fans (Type: Conexa JVF-550AX, $500 \mathrm{~W}$, nozzle diameter: $250 \mathrm{~mm}$, air flow: $0.69-0.97 \mathrm{~m}^{3} / \mathrm{s}$ ) are used in the tunnels area, with distance between the fans of about $83 \mathrm{~m}$. The CRT operates to transfer coal from the stockpile to a coal barge at the rate of 2000 tons of coal per hour. The CRT is in constant operation hence the cleaning of the CRT is difficult to conduct. Monitoring facilities are inadequate to check the concentration of methane and $\mathrm{CO}$ gas. The score for the risk assessment of the CRT in Mine A, as shown in Table 6, is 375.68, which is categorized as "High Risk".

The CRT in Mine B has the same dimensions and equipment as the CRT in Mine A. However, there are 12 jet fan units installed, with the distance between the fans about $21 \mathrm{~m}$. This CRT also operates to move coal from the stockpile to the coal barge at a rate of 2000 tons of coal per hour. The operation is not continuous, hence cleaning of the CRT is easily conducted. Monitoring facilities are inadequate to check the concentrations of methane and $\mathrm{CO}$ gas. The score for the risk assessment of the CRT in Mine B, as shown in Table 6 is 295.78, which is categorized as "Medium Risk".

AHP risk assessment results shows a good agreement with the site assessment, in that the CRT in Mine A is relatively unfavourable for safety conditions compared to Mine B. The difference between the scores is 79.9, which is relatively large and shows clear differences, especially related to the effectiveness of the ventilation conditions and coal production condition, which create larger amounts of methane and coal dust in Mine A than in Mine B.

Table 5 Comparison of the field measurement results in Mine A and Mine B

\begin{tabular}{|c|c|c|c|}
\hline No. & Parameters & Mine A & Mine B \\
\hline 1 & $\begin{array}{l}\text { Coal } \\
\text { spontaneous } \\
\text { combustion }\end{array}$ & $\begin{array}{l}\text { Average temperature for coal stored for } 1 \text { day in stockpiles } \\
\text { was } 31.8^{\circ} \mathrm{C} \text {, and for coal stored for } 21 \text { days was } \\
51.8^{\circ} \mathrm{C} \text {. There were also an indication of coal } \\
\text { spontaneous combustion in Mine A stockpiles }\end{array}$ & $\begin{array}{l}\text { Average temperature for coal stored for } 1-3 \text { days in } \\
\text { stockpiles was } 34.8^{\circ} \mathrm{C} \text {. The indication of coal } \\
\text { spontaneous combustion in Mine B stockpiles have not } \\
\text { found because the coal storing time is relatively short in } \\
\text { comparison with Mine A }\end{array}$ \\
\hline 2 & $\begin{array}{l}\text { Ventilation } \\
\text { system }\end{array}$ & $\begin{array}{l}\text { Average velocity on the CRT was } \\
\text { estimated: } 0.5-0.75 \mathrm{~m} / \mathrm{s}\end{array}$ & Average velocity on the CRT was estimated: $1-1.5 \mathrm{~m} / \mathrm{s}$ \\
\hline 3 & Coal dust & $\begin{array}{l}\text { There was an indication that coal dust cloud was } \\
\text { established in Mine A. Improvement of the coal dust } \\
\text { management will be needed to reduce the coal dust } \\
\text { explosion risk }\end{array}$ & $\begin{array}{l}\text { There was an indication that coal dust settled in floor, pipe } \\
\text { and steel near the coal chute gate. Coal dust cloud have } \\
\text { not found. That showed the ventilation system have been } \\
\text { diluted the coal flying-dust. However, the heavier coal } \\
\text { dust particle were settled down and need to be water } \\
\text { sprayed and managed regularly }\end{array}$ \\
\hline
\end{tabular}


Table 6 Risk assessment results of CRT in Mine A and Mine B

\begin{tabular}{|c|c|c|c|c|c|c|}
\hline \multirow[t]{2}{*}{ No. } & \multirow[t]{2}{*}{ Factor } & \multirow[t]{2}{*}{ Weighted value } & \multicolumn{2}{|c|}{ Mine A } & \multicolumn{2}{|c|}{ Mine B } \\
\hline & & & Value & Risk rating & Value & Risk rating \\
\hline 1 & Spontaneous combustion & 0.099 & 5 & 49.5 & 3 & 29.7 \\
\hline 2 & Normal air & 0.0825 & 5 & 41.25 & 5 & 41.25 \\
\hline 3 & Total resistance & 0.0569 & 4 & 85.76 & 2 & 42.88 \\
\hline 4 & Specifications of fan & 0.0538 & & & & \\
\hline 5 & Distance between fan & 0.0522 & & & & \\
\hline 6 & Number of fan & 0.0515 & & & & \\
\hline 7 & Monitoring facilities & 0.0468 & 5 & 23.4 & 5 & 23.4 \\
\hline 8 & Monitoring procedures & 0.0262 & 4 & 10.48 & 3 & 7.86 \\
\hline 9 & Unstable conditions around the tunnel & 0.0378 & 2 & 7.56 & 2 & 7.56 \\
\hline 10 & Coal properties & 0.0365 & 3 & 10.95 & 3 & 10.95 \\
\hline 11 & Coal production level (related to methane concentration in CRT) & 0.0355 & 5 & 17.75 & 4 & 14.2 \\
\hline 12 & Time period of coal in stockpile & 0.0222 & 4 & 8.88 & 3 & 6.66 \\
\hline 13 & Humidity & 0.0315 & 2 & 6.3 & 2 & 6.3 \\
\hline 14 & Addition of inert gases & 0.0275 & 2 & 5.5 & 2 & 5.5 \\
\hline 15 & Gases & 0.0248 & 2 & 4.96 & 2 & 4.96 \\
\hline 16 & Fire & 0.0206 & 4 & 8.24 & 4 & 8.24 \\
\hline 17 & Coal production level (related to coal dust concentration in CRT) & 0.0199 & 5 & 9.95 & 4 & 7.96 \\
\hline 18 & Design of tunnel & 0.0189 & 2 & 3.78 & 2 & 3.78 \\
\hline 19 & Total moisture & 0.0187 & 3 & 5.61 & 3 & 5.61 \\
\hline 20 & Cable systems & 0.0182 & 2 & 3.64 & 2 & 3.64 \\
\hline 21 & Size of void & 0.0174 & 4 & 6.96 & 4 & 6.96 \\
\hline 22 & Friction & 0.0165 & 3 & 4.95 & 3 & 4.95 \\
\hline 23 & Monitors layout & 0.0139 & 4 & 5.56 & 3 & 4.17 \\
\hline 24 & Monitoring staff & 0.0131 & 4 & 5.24 & 3 & 3.93 \\
\hline 25 & Volatile matter & 0.0128 & 4 & 5.12 & 4 & 5.12 \\
\hline 26 & Fireproof & 0.0128 & 4 & 5.12 & 4 & 5.12 \\
\hline 27 & Heat & 0.0124 & 3 & 3.72 & 3 & 3.72 \\
\hline 28 & Number of void & 0.0096 & 4 & 3.84 & 4 & 3.84 \\
\hline 29 & Electric motors at conveyor belt & 0.0091 & 3 & 2.73 & 3 & 2.73 \\
\hline 30 & Dust particle coagulation due to $\mathrm{RH}$ & 0.0089 & 2 & 1.78 & 2 & 1.78 \\
\hline 31 & Monitoring tools & 0.0069 & 4 & 2.76 & 4 & 2.76 \\
\hline 32 & Combustion & 0.0069 & 5 & 3.45 & 1 & 0.69 \\
\hline 33 & Ash content & 0.0064 & 4 & 2.56 & 4 & 2.56 \\
\hline 34 & Coal size in stockpile & 0.0061 & 4 & 2.44 & 4 & 2.44 \\
\hline 35 & Ambient temperature & 0.005 & 3 & 1.5 & 3 & 1.5 \\
\hline 36 & Electric motors at fan & 0.0049 & 2 & 0.98 & 2 & 0.98 \\
\hline 37 & Flame temperature level & 0.0047 & 2 & 0.94 & 2 & 0.94 \\
\hline 38 & Time to extinction & 0.0047 & 2 & 0.94 & 2 & 0.94 \\
\hline 39 & Type of agent (used in fire extinguishment) & 0.0047 & 2 & 0.94 & 2 & 0.94 \\
\hline 40 & Existence of winds & 0.0047 & 2 & 0.94 & 2 & 0.94 \\
\hline 41 & Barometric pressure & 0.0045 & 2 & 0.9 & 2 & 0.9 \\
\hline 42 & Pressure & 0.0041 & 2 & 0.82 & 2 & 0.82 \\
\hline 43 & Sulfur & 0.0038 & 4 & 1.52 & 4 & 1.52 \\
\hline 44 & Static electricity & 0.0032 & 2 & 0.64 & 2 & 0.64 \\
\hline 45 & Position of void & 0.003 & 4 & 1.2 & 4 & 1.2 \\
\hline 46 & Exhaust fan & 0.0022 & 4 & 0.88 & 4 & 0.88 \\
\hline 47 & Electronic devices & 0.0021 & 2 & 0.42 & 2 & 0.42 \\
\hline
\end{tabular}


Table 6 continued

\begin{tabular}{|c|c|c|c|c|c|c|}
\hline \multirow[t]{2}{*}{ No. } & \multirow[t]{2}{*}{ Factor } & \multirow[t]{2}{*}{ Weighted value } & \multicolumn{2}{|c|}{ Mine A } & \multicolumn{2}{|c|}{ Mine B } \\
\hline & & & Value & Risk rating & Value & Risk rating \\
\hline 48 & Type of fluid (used in dust spraying) & 0.0017 & 2 & 0.34 & 2 & 0.34 \\
\hline 49 & Non fireproof & 0.0014 & 4 & 0.56 & 4 & 0.56 \\
\hline 50 & Spraying pressure & 0.0004 & 2 & 0.08 & 2 & 0.08 \\
\hline 51 & Type of nozzle & 0.0002 & 2 & 0.04 & 2 & 0.04 \\
\hline \multirow[t]{2}{*}{52} & Diffusion coefficient & 0.0046 & 5 & 2.3 & 2 & 0.92 \\
\hline & Total risk rating & & & 375.68 & & 295.78 \\
\hline
\end{tabular}

Table 7 Risk assessment on CRT in Mine A and Mine B using checklist method

\begin{tabular}{|c|c|c|c|c|}
\hline \multirow[t]{2}{*}{ No. } & \multirow[t]{2}{*}{ Factors that affect CRT explosion } & \multicolumn{2}{|l|}{ Site conditions } & \multirow[t]{2}{*}{ Explanation } \\
\hline & & CRT in Mine A & CRT in Mine B & \\
\hline 1 & Fuel & & & \\
\hline 1.1 & Combustible gases & $\mathrm{V}$ & $\mathrm{V}$ & \\
\hline 1.2 & Dust & $\mathrm{V}$ & $\mathrm{V}$ & $\begin{array}{l}\text { Dust concentration in the CRT in Mine } A \text { is } \\
\text { higher than that in Mine B }\end{array}$ \\
\hline 1.3 & Methane & $\mathrm{V}$ & $\mathrm{V}$ & $\begin{array}{l}\text { Methane concentration in the CRT in Mine A is } \\
\text { higher than that in Mine B }\end{array}$ \\
\hline 2 & Oxygen & & & \\
\hline 2.1 & Planned of air & $\mathrm{V}$ & $\mathrm{V}$ & \\
\hline 2.2 & Unplanned of air & $\mathrm{V}$ & $\mathrm{V}$ & \\
\hline 3 & Ignition & & & \\
\hline 3.1 & Electricity & $\mathrm{V}$ & $\mathrm{V}$ & \\
\hline 3.2 & Chemically & $\mathrm{V}$ & $\mathrm{V}$ & \\
\hline 3.3 & Physically & $\mathrm{V}$ & $\mathrm{V}$ & \\
\hline 4 & Confinement & & & \\
\hline 4.1 & Design of tunnel & $\mathrm{V}$ & $\mathrm{V}$ & \\
\hline 4.2 & Unstable condition & $\mathrm{V}$ & $\mathrm{V}$ & \\
\hline 5 & Dispersion & & & \\
\hline 5.1 & Ventilation system & $\mathrm{V}$ & $\mathrm{V}$ & $\begin{array}{l}\text { The ventilation system of the CRT in Mine B is } \\
\text { more efficient than that in Mine A }\end{array}$ \\
\hline 5.2 & Diffusion coefficient & $\mathrm{V}$ & $\mathrm{V}$ & \\
\hline 5.3 & Design of tunnel & $\mathrm{V}$ & $\mathrm{V}$ & \\
\hline 6 & Monitoring system & & & \\
\hline 6.1 & Monitoring tools & $\mathrm{V}$ & $\mathrm{V}$ & \\
\hline 6.2 & Monitoring facilities & $\mathrm{V}$ & $\mathrm{V}$ & $\begin{array}{l}\text { Monitoring system in Mine B is } \\
\text { more sufficient than that in Mine A }\end{array}$ \\
\hline 6.3 & Monitoring staff & $\mathrm{V}$ & $\mathrm{V}$ & \\
\hline 6.4 & Monitoring procedures & $\mathrm{V}$ & $\mathrm{V}$ & \\
\hline 6.5 & Monitors layout & $\mathrm{V}$ & $\mathrm{V}$ & \\
\hline
\end{tabular}

\subsection{Explosion risk assessment of CRT in Mine $A$ and Mine B using checklist method}

To assess the effectiveness of the AHP method, the checklist method was used to compare CRT conditions in Mine A and Mine B. The checklist method has been used in practice by engineers to observe the safety conditions of working areas such as CRTs. The checklist method is a deductive technique derived from the risks encountered previously and provides a convenient means for management to rapidly identify possible risks by using either a series of questions or a list of topics to be considered (Merna and Al-Thani 2008).

Table 7 shows a risk assessment performed using the checklist method to assess the risk of explosion in CRT. The factors observed in the checklist referred to the 
structure of AHP especially in the third hierarchy (Fig. 4), which can be directly observed in the CRT facility. From the analysis, the checklist shows that conditions in both CRTs have the same risk of the explosion. In addition, the CRT in Mine A is generally less safe than the CRT in Mine $\mathrm{B}$, which can be distinguished qualitatively by professional judgement as shown in the explanation column in Table 7. The checklist method can identify the potential hazards and the degree of risk qualitatively, however, the method cannot identify important factors which have to be taken into account to reduce the explosion risk levels and that can be quantified in the developed AHP method.

\section{Concluding remarks}

The modified analytical hierarchy process (AHP) method can be applied to describe and explain quantitatively the factors which have caused explosion incidents in coal reclaim tunnels (CRT)We have listed 6 main factors (main criteria) and 52 sub-factors related to the incidents in CRT. Among all these factors, the most contributing factors on the occurrence of explosion in CRT are drawn as follows:

(1) Mechanical Ventilation, consisting of total resistance, fan specifications, distance between fans and number of fans, with total weighted value of 0.2144 ;

(2) Monitoring System, consisting of monitoring facilities, monitoring procedures, monitor layout, monitoring staff, and monitoring tools, with total weighted value of 0.1081 ; and

(3) Coal Spontaneous Combustion, with weighted value of 0.0990 .

These main contributing factors must be taken into account in order to minimize the CRT's risk of explosion.

The practice of these risk criteria to assess CRT explosion risk should be carefully investigated further to be certain for the risk classification, especially in determining "the cause factors", "the factor's weighted value", and "the interpretation of risk classification", that possibly very specific for each CRT area and situation. In the future, research must continue and develop these factors and their measurement, especially in re-assessing and quantifying all of the AHP factors, along with conducting assessments of several CRTs of different types and operational conditions.

\footnotetext{
Acknowledgements Authors gratefully acknowledge Institut Teknologi Bandung, Indonesia, under "Program Penelitian, Pengabdian kepada Masyarakat, dan Inovasi (P3MI) ITB" research grant 2017 and "Program Penelitian Luar Negeri ITB" research grant 2016, for financially support to the research. Authors would like to acknowledge Institut Teknologi Bandung and Kyushu University for supporting the research facilities. Authors would like to thanks to Prof. Rudy Sayoga Gautama, Prof. Made Astawa Rai, Simon H.
}

Prassetyo, Ph.D. of Institut Teknologi Bandung, Indonesia, and Prof. Kyuro Sasaki in Kyushu University, Japan for valuable suggestions to the research.

Open Access This article is distributed under the terms of the Creative Commons Attribution 4.0 International License (http://crea tivecommons.org/licenses/by/4.0/), which permits unrestricted use, distribution, and reproduction in any medium, provided you give appropriate credit to the original author(s) and the source, provide a link to the Creative Commons license, and indicate if changes were made.

\section{References}

Aristien HDP, Widodo NP (2015) Modeling of coal temperature distribution to estimate rate of temperature increase on coal stockpile spontaneous combustion using finite difference method. In: Proceedings of the international symposium on earth science and technology

Beamish BB, Arisoy A (2008) Effect of intrinsic coal properties on self-heating rates. In: Proceedings of the 12th US/North American mine ventilation symposium, pp 149-153

Beamish BB, Hamilton GR (2005) Effect of moisture content on the R 70 self-heating rate of Callide coal. Int J Coal Geol 64(1):133-138

Beamish BB, Barakat MA, St George JD (2000) Adiabatic testing procedures for determining the self-heating propensity of coal and sample ageing effects. Thermochim Acta 362(1-2):79-87

Beamish BB, Barakat MA, George JDS (2001) Spontaneouscombustion propensity of New Zealand coals under adiabatic conditions. Int J Coal Geol 45(2-3):217-224

Bill Adams \& the Creative Decision Foundation (2016) Super decision software

Brnich MJ, Kowalski-Trakofler KM (2010) Underground coal mine disasters 1900-2010: events, responses, and a look to the future. In: Extracting the science: a century of mining research, pp 363-372

Brooks K, Svanas N, Glasser D (1988) Evaluating the risk of spontaneous combustion in coal stockpiles. Fuel 67(5):651-656

Brune JF, Cashdollar KL, Zipf RK (2007) Explosion prevention in United States coal mines. In: Proceedings of the 32nd international conference of safety in mines research institutes, pp 1-7

Chalmers DR (2013) Explosions in mines-systematic failure. In: 23rd World mining congress 2013 proceedings, Montreal

Coward HF, Jones GW (1952) Limits of flammability of gases and vapors (No. BM-BULL-503). Bureau of Mines, Washington

Denton (2004) The prevention and control of explosion in mines. The Mining Association of the United Kingdom, London

Feng KK, Chakravorty RN, Cochrane TS (1973) Spontaneous combustion a coal mining hazard. Can Min Metall (CIM) Bull UK 66:75-84

Guidelines MDG 28-Technical Reference (2013) Safety requirements for coal stockpiles and reclaim tunnel. Produced by Mine Safety Operation Branch Industry and Investment NSW

Guidelines MDG 1006-Technical Reference (2011) Technical reference for spontaneous combustion management guidelines. Produced by Mine Safety Operation Branch Industry and Investment NSW

Humphreys D, Rowlands D, Cudmore JF (1981) Spontaneous combustion of some Queensland coals. In Proceedings of ignitions, explosions and fires in coal mines symposium. pp 5-1

Iqbal M (2016) Study of mixing hot and cold air in underground tunnel in laboratory scale. Final Project, Department of Mining 
Engineering, Institut Teknologi Bandung. Unpublished manuscript

Juanzah A (2017) Study on distribution of methane gas for coal reclaim tunnel Kintap site PT. Arutmin Indonesia, in laboratory scale. Final Project, Department of Mining Engineering, Institut Teknologi Bandung. Unpublished manuscript

Kim AG (1977) Estimating methane content of bituminous coalbeds from adsorption data. Report of Investigation 8245, U.S. Bureau of Mines, Washington

Kissell FN, Tien JC, Thimons ED (2007) Methods for controlling explosion risk at coal mine working faces. In: Proceeding of the 32nd international conference of safety in mines research institutes, Beijing, pp 161-168

Kusuma RP (2016a) Analysis of heat transfer in mining ventilation network using physical model at laboratory scale and software ventsim visual 4 simulations. Final Project, Department of Mining Engineering, Institut Teknologi Bandung. Unpublished manuscript

Kusuma BJ (2016b) Modeling analysis on distribution of methane gas for straight lines and turn lines flow of longwall mining using CFD software in laboratory scale. Final Project, Department of Mining Engineering, Institut Teknologi Bandung. Unpublished manuscript

Lang L, Fu-Bao Z (2010) A comprehensive hazard evaluation system for spontaneous combustion of coal in underground mining. Int $\mathrm{J}$ Coal Geol 82(1):27-36

McPherson MJ (2012) Subsurface ventilation and environmental engineering. Springer, Berlin

Merna T, Al-Thani FF (2008) Corporate risk management. Wiley, Berlin
Nalbandian H (2010) Propensity of coal to self-heat. IEA Clean Coal Centre, London, p 47

Pratama A (2014) Study of coal spontaneous combustion in PTBATanjung Enim stockpile. Final Project, Department of Mining Engineering, Institut Teknologi Bandung. Unpublished manuscript

Pratama BM (2016) Analysis on distribution of methane gas in coal longwall mining using laboratory scale model. Final Project, Department of Mining Engineering, Institut Teknologi Bandung. Unpublished manuscript

Ren TX, Edwards JS, Clarke D (1999) Adiabatic oxidation study on the propensity of pulverized coals to spontaneous combustion. Fuel 78(14):1611-1620

Ristić D (2013) A tool for risk assessment. Inženjerstvo Zaštite 3:121

Saaty TL (1980) The analytic hierarchy process: planning, priority setting, and resource allocation. McGraw-Hill, New York

Saaty TL (2008) Decision making with the analytic hierarchy process. Int J Serv Sci 1(1):83-98

Sensogut C, Cinar I (2006) A research on the spontaneous combustion tendency of Turkish coals-ilgin lignite's case. Trends Appl Sci Res 1:9-14

Smith GL, Du Plessis JJL (1999) Control strategies for coal dust and methane explosions in underground coal mines: current South African research and development initiatives

Stephan CR (1998) Coal dust explosion hazards. Preprints-Society of Mining Engineers of AIME, New York

Uludag S (2007) A visit to the research on Wits-Ehac index and its relationship to inherent coal properties for Witbank Coalfield. J South Afr Inst Min Metall 107:671-679

Yuan L, Smith AC (2012) The effect of ventilation on spontaneous heating of coal. J Loss Prev Process Ind 25(1):131-137 